\title{
Modelling of droplet impacts on dry and wet surfaces using depth-averaged form
}

\author{
Krish S. L. Hook • Sergii Veremieiev $\mathbb{D}$
}

Received: 11 May 2020 / Accepted: 5 December 2020 / Published online: 15 February 2021

(C) The Author(s) 2021

\begin{abstract}
An efficient time-adaptive multigrid algorithm is used to solve a range of normal and oblique droplet impacts on dry surfaces and liquid films using the Depth-Averaged Form (DAF) method of the governing unsteady Navier-Stokes equations. The dynamics of a moving three-phase contact line on dry surfaces is predicted by a precursor film model. The method is validated against a variety of experimental results for droplet impacts, looking at factors such as crown height and diameter, spreading diameter and splashing for a range of Weber, Reynolds and Froude numbers along with liquid film thicknesses and impact angles. It is found that, while being a computationally inexpensive methodology, the DAF method produces accurate predictions of the crown and spreading diameters as well as conditions for splash, however, underpredicts the crown height as the vertical inertia is not included in the model.
\end{abstract}

Keywords Adaptive time-stepping · Crown formation · Depth-averaged form · Droplet impact · Liquid films . Splashing

\section{Introduction}

Droplet impacts appear in a variety of engineering applications that would benefit from an improved understanding of the droplet-film interactions, allowing for optimisation of the impact behaviour for any particular situation. Often, a droplet will be impacting upon a dry surface which quickly changes into a thin liquid film created by deposition of the previous droplets [1]. This is the case in spray painting, surface cleaning and ink-jet printing where achieving a large droplet spread without splashing is desirable [2]. For the case of fuel injection within internal combustion engines, splashing may be desirable to create secondary atomisation and increase the fuel vaporisation rate. However, this may also create deviancies in fuel-air equivalencies which influence heat transfer and are not incorporated into design calculations [3].

Due to the complexity of interactions between multiple droplets, it is difficult to create an accurate numerical simulation of droplet interactions [4]. An abundance of research has been carried out to validate that the behaviour

K. S. L. Hook · S. Veremieiev $(\varangle)$

Department of Engineering, Durham University, Lower Mount Joy, South Road, Durham DH1 3LE, UK

e-mail: s.veremieiev@durham.ac.uk

K. S. L. Hook

e-mail: krishhook@hotmail.co.uk 
of a single drop can be extrapolated to explain how the whole spray will behave, and determine the maximum spray density to make this a valid assumption [5,6]. In most practical situations, as in this study, it is accepted that the droplets are distant enough from each other such that the whole spray can be represented as a single impact.

The dynamics of an impact, and consequent action of the fluid, depend upon a variety of fluid properties and other physical factors [7]. On dry surfaces, droplets may disperse across the surface in the form of secondary droplets or by spreading. The rate at which they spread and the equilibrium state depends strongly upon the contact angle, which varies according to the surface wettability, whether the droplet is advancing or receding and the conditions of impact [8]. How these parameters influence each other is not understood in detail and models vary accordingly [9-11]. When striking a liquid film, on a macroscopic level, the impact dynamics tend to be more complex as a crown will generally form, accompanied by prompt and secondary splashing [12]. After initial crown formation and splashing, capillary waves then propagate away from the site of the impact.

The earliest studies of droplet impacts used high-speed photography to capture the action of the fluid. Worthington and Cole carried out experiments in the late 19th century to capture snapshots of the splashing mechanism, collating some of the first qualitative data of splashing and crown formation [13]. Their studies looked at the effects of liquid properties upon the splash but had little consideration of film thickness and other physical factors. It was not until the late 20th century that the characteristics of droplet impact were linked to physical factors such as surface roughness and film thickness [14].

These developments were largely due to rapid development of experimental techniques and high-speed cameras, where more studies obtained high-quality images of splashing. Using these techniques, Vander Wal et al. and Cossali et al. investigated droplets of different liquids splashing on films of various depths, producing a trove of data documenting splashing profiles under different conditions $[6,15]$. Rioboo et al. experimented with a variety of conditions to determine the nature of crown formation, finding their results could all be explained as a combination of the Weber number, Ohnesorge number and a dimensionless film thickness [16]. Rioboo et al. and Šikalo et al. conducted further similar broad-ranging experiments for dry surface impacts, looking at how spreading factors, contact line velocities and contact angles varied for different liquids and impact conditions $[8,11,17,18]$.

Macklin and Mexas produced the first numerical model describing the effect of film thickness upon crown shape [19]. This was one of the first uses of the dimensionless Weber, Reynolds, and Froude numbers, which have since been used regularly in this field. Yarin and Weiss then proposed a breakthrough model of a kinematic discontinuity across the crown itself, predicting splashing as a complicated function of density, viscosity, surface tension and drop frequency [1]. Other studies then used this model to develop analytical solutions of crown shape and dynamic equations of motion for droplet impacts [4,20,21]. Investigations such as Trujillo and Lee generalised Yarin and Weiss' theory, determining the effects of film thickness, wall friction and impact angle upon the crown dynamics [22]. Yarin and Weiss then produced a further study of a single drop impact, obtaining a direct numerical simulation of the velocity field, using a boundary-integral method based on scalar velocity potential [23]. This produced results similar to their previous experiments, and from this work, Cossali et al. produced empirically derived constants which could be used to model the crown's growth radially [24].

For dry impacts, studies have attempted to predict the dynamic contact angle, spreading diameter, splashing, deposition and other physical outcomes [25-27]. Predictions of the dynamic contact angle and maximum spreading diameter used either completely empirical or semi-empirical models [27,28]. As no single model has been consolidated for the dynamic contact angle, various models are still relevant [10]. Past models for spreading diameters were based on the energy balance of the drop and assumed the drop shape to be a disc at equilibrium [29]. Roisman et al. discovered that the droplet impact is more complex than this and accounted for the effects of inertial, viscous and surface forces, as well as wettability in their model [30].

Various methods have been used to numerically solve droplet impacts such as; Volume of Fluid (VOF), Level Set, Combined Level Set, Level Contour Reconstruction methods, and a two-phase flow Lattice Boltzmann model [3134]. VOF, which is based upon the Navier-Stokes (N-S) equations, has been used to develop velocity and pressure fields within a droplet impact and investigate the effects of surrounding gas density [35]. Rieber and Frohn used VOF to show a Rayleigh instability as the reason for secondary splashes during disintegration of the splashing lamella, 
though this was disputed by Krechetnikov and Homsy, who instead suggested Richtymer-Meshkov instabilities $[21,36]$.

The Depth-Averaged Form (DAF) method used in this study is often used in modelling thin-film liquid flow to determine the concourse and velocity of rivers [37-39]. The DAF method can be thought of as a first-order accurate long-wave approximation with no Reynolds number limitation. Similar to VOF, this is based upon the N-S equations, and is being used after Veremieiev et al. used an efficient strategy to solve the governing DAF, paving the way for its applicability to droplet impacts [38]. Veremieiev has used this approach repeatedly, to investigate both electrified thin-film flow and droplets of bio-pesticide flowing over foliage [40,41]. The method of depth-averaging 2D N-S equations has also been referred to as the Integral Boundary Layer (IBL) approximation and has also been presented by Shkadov and Ruyer-Quil and Manneville [42-44].

In this study, there are 2D axisymmetric, 3D axisymmetric and 3D oblique simulations that are carried out for a variety of fluids, all of which are directly compared with experimental data from Vander Wal et al., Cossali et al. and Šikalo et al. $[6,8,11,15]$. This allows for a robust examination of the DAF method, as multiple parameters are considered and the accuracy of each determined.

Due to the computational demands of simulations, two main techniques are used to make the solver used more efficient; adaptive time-stepping and a multigrid solution. Adaptive time-stepping enables the algorithm used for the solution to be optimised in a controlled manner, subject to a specific error tolerance [45]. A multigrid solution, rather than reducing all errors on the same fine grid, which can be unacceptable in terms of computational time, will change to a coarse grid to reduce low frequency errors [46]. Different methods have been used to input both these techniques into various algorithms, increasing the efficiency of many computationally-demanding problems, of which droplet impact modelling is included [47-49].

The adaptive time-stepping scheme used in this algorithm is based on Heun's second-order predictor-corrector method [45,47], which automatically adjusts the time step subject to the error tolerance, allowing it to optimise. Multigrid methods use a smoother on a sequence of coarser computational grids to reduce high frequency error components on a particular grid $[46,48,49]$. They are often used to solve the discrete analogues within a wide variety of flow problems where advancements in efficiency and resources are being made [50]. This study combines both adaptive time-stepping and the multigrid method to reduce the computational resource required for the calculations as would otherwise be required. All impact simulations are modelled using $\mathrm{C}++$ and run within the Linux-based Hamilton cluster environment.

The underlying theoretical concepts defining the droplet impact and the DAF method of the governing N-S equations are presented in Sect. 2, along with the conditions used for the various simulations carried out. Section 3 describes the numerical conditions used for the solution method, including the spatial discretisation of the impact domain and temporal discretisation used for the solution. In Sect. 4, a comparison between the theoretical predictions of the model and experimental data is undertaken. These discussions first establish the accuracy of the DAF method for modelling droplet impacts, before identifying the parameters that most affect behaviours and outcomes of impacts. Finally, conclusions drawn from the study are outlined in Sect. 5.

\section{Problem formulation}

Figure 1 shows a schematic of a droplet impacting upon a film in two stages. The liquid is assumed to be incompressible with constant density $\rho$, dynamic viscosity $\mu$, and surface tension $\sigma$. For the droplet simulations the Cartesian $X, Y, Z$ coordinate system is adopted, with the solution domain bounded vertically at the bottom of the liquid film where $Z=0$, and from above by the free surface $Z=H(X, Y, T)$, where $T$ is time and $H$ is the height of the free surface above the solid impact plane. The domain is also bounded horizontally, with a length $L$ in the $X$ plane and a width $W$ in the $Y$ plane, where the origins of both the $X$ and $Y$ axes are located at the centre of each plane.

Stage (1) in Fig. 1a shows the droplet with an initial in-flight diameter $D$ and initial velocity $\mathbf{U}_{0}=\left(U_{\mathrm{t}}, 0,-U_{0}\right)$, where $U_{0}$ and $U_{\mathrm{t}}$ are the normal and tangential velocity components, respectively. The droplet also has an angle of impact $\theta$, where $\tan \theta=U_{\mathrm{t}} / U_{0}$ and is accelerating within a normal gravitational field $\mathbf{g}=\left(0,0,-g_{0}\right)$, where 

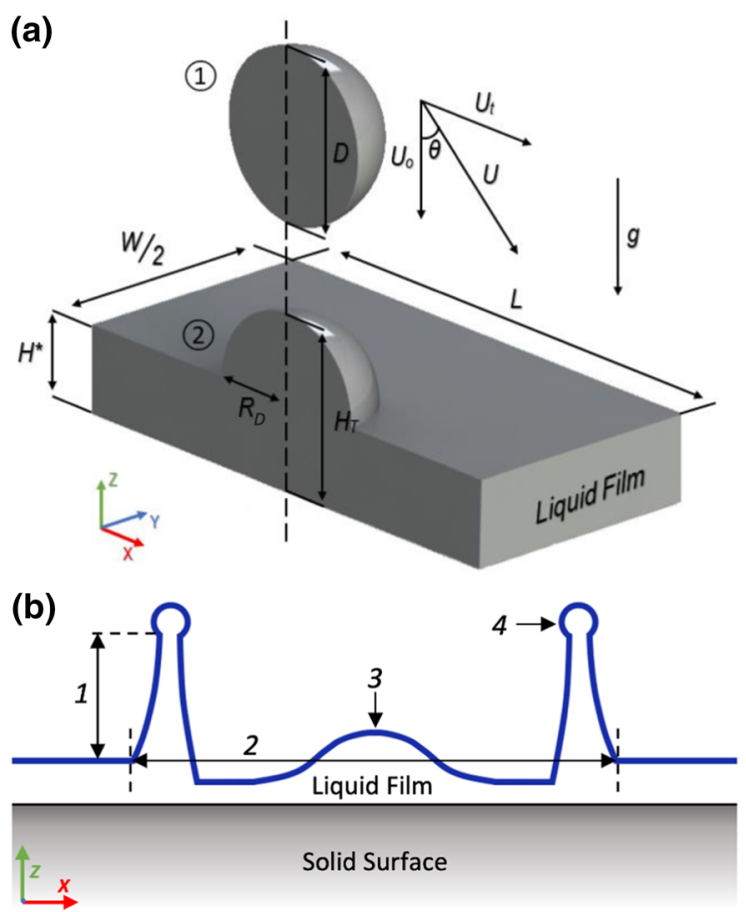

Fig. 1 Schematic of droplet impact; a Stage (1) shows the droplet before impact, with Stage (2) showing the droplet at initial contact with the liquid film where it is modelled as a spherical cap, $\mathbf{b}$ shows the formation of a crown after impact where $\mathbf{1}$ is the crown height, $\mathbf{2}$ is the lower external crown diameter, $\mathbf{3}$ is the residual shape of the impacting drop and $\mathbf{4}$ is the cross-section of the free rim

$g_{0}=9.81 \mathrm{~ms}^{-2}$ is the acceleration due to gravity. When $\theta=0$ a normal impact takes place and when $\theta>0$, the impact is oblique. The initial volume of the droplet is $V_{0}=(1 / 6) \pi D^{3}$.

In Stage (2), the droplet has impacted with a liquid film of thickness $H^{*}$ and the initial droplet impact site has an in-plane radius of $R_{\mathrm{D}}$. The total initial height of the film and droplet is $H_{T}$ and the initial droplet height upon impact is $H_{D}$, where $H_{T}=H_{D}+H^{*}$. Upon impact, the droplet is modelled as a spherical cap with volume $V=\frac{\pi}{3} H_{D}^{2}\left(3 R_{\mathrm{D}}-H_{D}\right)$. Once the droplet is in contact with the film, they are considered as one volume of fluid. Immediately after impact, a crown of height $H$, which is a priori unknown, will form around the impact site and propagate away, capillary waves will also form and fingering or splashing may occur.

Figure $1 \mathrm{~b}$ shows formation of a crown after impact using the same Cartesian $X, Y, Z$ coordinate system. Crown height, shown as $\mathbf{1}$, is the distance between the liquid film and the bottom of the free rim, shown as $\mathbf{4}$, which is unstable liquid at the top of the crown from which secondary droplets may form. Crown diameter, shown as $\mathbf{2}$, is taken to be the lower external diameter of the crown, and both the crown diameter and height are defined to be consistent with [15].

\subsection{Governing equations}

For comparison between the results of this study and experimental data, dimensionless variables are used both to define the simulation model and in the analysis. The variables that define the droplet impact model are transformed into dimensionless scales and are shown below:

$\left(x, y, z, l, w, r, d, h, r_{\mathrm{d}}, h_{\mathrm{d}}\right)=\frac{\left(X, Y, Z, L, W, R, D, H, R_{\mathrm{D}}, H_{\mathrm{D}}\right)}{D},(u, v, w)=\frac{(U, V, W)}{U_{0}}, \quad t=\frac{T U_{0}}{D}, \quad p=\frac{D P}{\sigma}$. 
From Eq. (1), $\mathbf{U}=(U, V, W)$ and $p$ are the velocity and pressure within the fluid and $\left(X, Y, Z, L, W, R, D, H, R_{\mathrm{D}}, H_{\mathrm{D}}, U, V, W, T, P\right) \rightarrow\left(x, y, z, l, w, r, d, h, r_{\mathrm{d}}, h_{\mathrm{d}}, u, v, w, t, p\right)$ represent the change between dimensional and dimensionless variables, respectively.

The three dimensionless groups governing the droplet impact and its flow regime are defined below:

$\operatorname{Re}=\frac{\rho U_{0} D}{\mu}, \quad$ We $=\frac{\rho U_{0}^{2} D}{\sigma}, \quad$ Fr $=\frac{U_{0}}{\sqrt{g_{0} D}}$.

The Reynolds number Re is the ratio of inertia to viscous forces within the fluid, the Weber number We is the ratio of inertia to surface tension forces and the Froude number Fr is a measure of the inertia with respect to gravitational forces upon the fluid flow. Comparatively, Fr is not as influential as Re and We in most practical situations as the effects of $\mathbf{g}$ upon small volumes of fluid is minimal. However, as velocity decreases and the film becomes thicker, the effect is no longer negligible; hence, Fr has been considered in this study.

The incompressible liquid flow of the impact is described by the $\mathrm{N}-\mathrm{S}$ and continuity equations, conserving momentum and mass, respectively, shown in dimensionless form:

$$
\begin{aligned}
& \operatorname{Re}\left(\frac{\partial \mathbf{u}}{\partial t}+\mathbf{u} \cdot \nabla \mathbf{u}\right)=-\frac{\operatorname{Re}}{\mathrm{We}} \nabla p+\nabla \boldsymbol{\tau}+\frac{\operatorname{Re}}{\operatorname{Fr}^{2}} \mathbf{g}, \\
& \nabla \cdot \mathbf{u}=0,
\end{aligned}
$$

where $\boldsymbol{\tau}=\nabla u+(\nabla u)^{\mathrm{T}}$ is the viscous stress tensor and $\nabla=(\partial / \partial x, \partial / \partial y, \partial / \partial z)$ is a vector operator nabla. The problem is then fully defined with the imposition of the required no-slip, outflow, kinematic, free-surface normal and tangential stress boundary conditions:

$$
\begin{aligned}
& \left.\mathbf{u}\right|_{z=0}=0, \\
& \frac{\partial h}{\partial t}+\left.u\right|_{z=h} \frac{\partial h}{\partial x}+\left.v\right|_{z=h} \frac{\partial h}{\partial y}-\left.w\right|_{z=h}=0, \\
& -\left.p\right|_{z=h}+\left(\left.\tau\right|_{z=h} \cdot \mathbf{n}\right) \cdot \mathbf{n}=\frac{\operatorname{Re}}{\mathrm{We}} \kappa, \\
& \left(\left.\tau\right|_{z=h} \cdot \mathbf{n}\right) \cdot \mathbf{t}=0,
\end{aligned}
$$

where $\mathbf{n}=(-(\partial h / \partial x),-(\partial h / \partial y), 1) \cdot\left[(\partial h / \partial x)^{2}+(\partial h / \partial y)^{2}+1\right]^{-1 / 2}$ is the normal unit vector pointing out from the free surface, $\mathbf{t}=\left(\alpha_{\mathrm{t}}, \beta_{\mathrm{t}}, \alpha_{\mathrm{t}}(\partial h / \partial x)+\beta_{\mathrm{t}}(\partial h / \partial y)\right) \cdot\left[\alpha_{\mathrm{t}}^{2}+\beta_{\mathrm{t}}^{2}+\left(\alpha_{\mathrm{t}}(\partial h / \partial x)+\beta_{\mathrm{t}}(\partial h / \partial y)\right)^{2}\right]^{-1 / 2}$ is the unit vector tangential to the free surface, $\alpha_{\mathrm{t}}$ and $\beta_{\mathrm{t}}$ are variables that define the direction of the vector at a point in the tangent plane and $\kappa=-\nabla \cdot \mathbf{n}$ is twice the mean free-surface curvature.

Solving Eqs. (3) and (4) to the boundary conditions (5)-(8), although possible, is extremely challenging and would require a lot of computer resource and time in order to obtain a mesh-independent solution. To bypass this it is possible to use the long-wave approximation based on the assumption that the ratio $\mathscr{E}=H_{0} / L_{0} \ll 1$, showing that the characteristic length scale in the vertical direction $H_{0}$, is much smaller than the characteristic length scale in the horizontal direction $L_{0}$. In addition to aiding the theoretical predictions of the splash, this assumption effectively reduces the dimensionality of the problem by one. Using this assumption, the resulting depth-averaged form of Eqs. (3) and (4) retains the inertia terms necessary for modelling droplet impacts. The ratio of scales $H_{0} \ll L_{0}$ is true for most of the times of droplet impact (with the exception of the initial moments) and is used for the DAF derivation as presented in Appendices A and B and [38]. Please note, however, that for consistency with the previous studies and convenience of presenting results, the in-flight diameter is used as both the horizontal and vertical scale, such as $H_{0}=L_{0}=D$, in the main text:

$$
\begin{aligned}
& \operatorname{Re}\left[\frac{\partial \bar{u}}{\partial t}+\frac{\partial \bar{u}}{\partial x}+\frac{\partial \bar{u}}{\partial y}+\frac{1}{5 h}\left(h \bar{u} \frac{\partial \bar{u}}{\partial x}+h \bar{v} \frac{\partial \bar{u}}{\partial x}-\bar{u} \frac{\partial h}{\partial t}\right)\right]=-\frac{\operatorname{Re}}{\text { We }} \frac{\partial p}{\partial x}+\frac{3 \bar{u}}{h^{2}}, \\
& \operatorname{Re}\left[\frac{\partial \bar{v}}{\partial t}+\frac{\partial \bar{v}}{\partial x}+\frac{\partial \bar{v}}{\partial y}+\frac{1}{5 h}\left(h \bar{u} \frac{\partial \bar{v}}{\partial x}+h \bar{v} \frac{\partial \bar{v}}{\partial x}-\bar{v} \frac{\partial h}{\partial t}\right)\right]=-\frac{\operatorname{Re}}{\text { We }} \frac{\partial p}{\partial y}+\frac{3 \bar{v}}{h^{2}}, \\
& \frac{\partial h}{\partial t}+\frac{\partial}{\partial x}(h \bar{u})+\frac{\partial}{\partial y}(h \bar{v})=0,
\end{aligned}
$$




$$
p=-\frac{\mathrm{We}}{\mathrm{Fr}^{2}}\left(\frac{\partial^{2} h}{\partial x^{2}}+\frac{\partial^{2} h}{\partial y^{2}}\right)+\frac{\mathrm{We}}{\mathrm{Fr}^{2}}(h-z)-\frac{\mathrm{We}}{\mathrm{Fr}^{2}} \Pi(h),
$$

where the over-bar denotes the depth-averaged components of velocity as per Eq. (30).

The dynamics of droplet impacts on dry surfaces are modelled by a precursor film model [51] that assumes the presence of a thin uniform liquid layer of thickness $h^{*}$ preceding and surrounding the droplet and covering the substrate. The application of the precursor film removes the triple point present at the contact line, removing the stress singularity [52]. Experimental evidence suggests that the physical thickness of the film is extremely small, around 1-100 $\mathrm{nm}$ as per [53]. However, the spatial resolution used must be at least of a comparable scale to the precursor film thickness, and using a precursor film that is too large leads to a systematic overprediction of the spreading rate. A disjoining pressure is then introduced across the film at the free-surface condition (7), to account for long-range inter-molecular interactions at the interfaces between the gas, the solid and the liquid droplet and the effect of the static equilibrium contact angle $\theta_{\mathrm{e}}$, between the three phases:

$\Pi(h)=\frac{(n-1)(m-1)\left(1-\tan \theta_{\mathrm{e}}\right)}{2 h^{*}(n-m)} \cdot\left[\left(\frac{h^{*}}{h}\right)^{n}-\left(\frac{h^{*}}{h}\right)^{m}\right]$,

where $n$ and $m$ are constants representing the disjoining pressure attraction and repulsion, respectively, set to $n=6$ and $m=3$ for all trials in this study [54]. Note that for impacts on wet surfaces the disjoining pressure (13) is set to zero.

Equations (9)-(12) are solved subject to symmetry boundary conditions at the edges of the computational domain, namely:

$\left.\frac{\partial(\bar{u}, \bar{v}, p, h)}{\partial x}\right|_{x=0, l}=\left.\frac{\partial(\bar{u}, \bar{v}, p, h)}{\partial y}\right|_{y=0, w}=0$.

\subsection{Initial conditions}

The initial conditions of the droplet correspond to the moment the droplet impacts upon the liquid film or dry surface. Upon impact, the droplet is modelled as a spherical cap with in-plane radius $r_{\mathrm{d}}$, height above the film $h_{\mathrm{d}}$, and in-plane centre coordinates $x_{\mathrm{d}}$ and $y_{\mathrm{d}}$ :

$h(x, y)= \begin{cases}h^{*}+\frac{h_{\mathrm{d}}^{2}-r_{\mathrm{d}}^{2}}{2 h_{\mathrm{d}}}+\sqrt{\frac{\left(r_{\mathrm{d}}^{2}+h_{\mathrm{d}}^{2}\right)^{2}}{4 h_{\mathrm{d}}^{2}}-\left(x-x_{\mathrm{d}}\right)^{2}-\left(y-y_{\mathrm{d}}\right)^{2}} & \text { if }\left(x-x_{\mathrm{d}}\right)^{2}+\left(y-y_{\mathrm{d}}\right)^{2} \leq r_{\mathrm{d}}^{2}, \\ h^{*}, & \text { otherwise. }\end{cases}$

The relationship between $r_{\mathrm{d}}$ and $h_{\mathrm{d}}$ is determined via conservation of volume of the droplet before and after impact $V_{0}=V$, which gives

$h_{\mathrm{d}}^{3}+3 r_{\mathrm{d}}^{2} h_{\mathrm{d}}-1=0$.

The droplet contact angle can now be determined from Eq. (15) for a spherical cap, as follows:

$\left.\frac{\partial h}{\partial r}\right|_{\left(x-x_{\mathrm{d}}\right)^{2}+\left(y-y_{\mathrm{d}}\right)^{2}=r_{\mathrm{d}}^{2}}=-\frac{2 r_{\mathrm{d}} h_{\mathrm{d}}}{r_{\mathrm{d}}^{2}-h_{\mathrm{d}}^{2}}=-\tan \theta_{\mathrm{c}}$.

The relationship between the radius of a spherical cap $r_{\mathrm{d}}$ and the angle $\theta_{\mathrm{c}}$ at which it meets the surface (referred to as the contact angle for dry surfaces) is now determined by combining Eqs. (16) and (17) as follows:

$r_{\mathrm{d}}=\tan \theta_{\mathrm{c}}\left[4\left(1+\tan ^{2} \theta_{\mathrm{c}}\right)^{3 / 2}-4-6 \tan ^{2} \theta_{\mathrm{c}}\right]^{-1 / 3}$.

Note that Stage (1) in Fig. 1 cannot be modelled as the initial condition for DAF since the droplet free surface is multivalued. Therefore, the droplet is modelled starting at the earliest point, depicted as Stage (2) in Fig. 1, when $\theta_{\mathrm{c}}=90^{\circ}$ and $h_{\mathrm{d}}=r_{\mathrm{d}}=2^{-2 / 3}=0.6300$. Please note for good comparison between the DAF predictions and experimental data, time-shifts, denoted by $t_{\mathrm{s}}$, are applied that correspond to how long the droplet in the experimental data takes to reach $d\left(t_{\mathrm{s}}\right)=2 r_{\mathrm{d}}=1.2600$ and $h\left(t_{\mathrm{s}}\right)=h_{\mathrm{d}}=0.6300$. 
Table 1 Fluid properties

\begin{tabular}{llcllll}
\hline & $D(\mathrm{~mm})$ & $\rho\left(\mathrm{kg} / \mathrm{m}^{3}\right)$ & $\sigma(\mathrm{N} / \mathrm{m})$ & $\mu(\operatorname{Pa~s})$ & $\theta_{\mathrm{e}}\left({ }^{\circ}\right)$ & $u_{o}(\mathrm{Dry})$ \\
\hline Hexadecane [6] & 2.00 & 773 & 0.0271 & 0.00340 & 11.2 & 0.31 \\
85\% glycerin [8] & 2.45 & 1220 & 0.0630 & 0.11600 & 63.8 & 0.02 \\
Isopropanol [11] & 1.80 & 786 & 0.0210 & 0.00240 & 6.5 & 0.40 \\
Water [15] & 3.82 & 1000 & 0.0728 & 0.00098 & - & - \\
\hline
\end{tabular}

Following [1], the kinematic discontinuity at the contact line is taken as the initial condition for averaged velocities $\bar{u}$ and $\bar{v}$. For axisymmetric impacts only one component, $\bar{u}$, is required. Oblique impacts require consideration of a tangential velocity, $u_{\mathrm{t}}$, and an impact angle, $\theta$ :

$$
\begin{aligned}
& \bar{u}(x, y)= \begin{cases}\frac{u_{0}\left(x-x_{\mathrm{d}}\right)}{r_{\mathrm{d}}}+u_{\mathrm{t}} \cos \theta & \text { if }\left(x-x_{\mathrm{d}}\right)^{2}+\left(y-y_{\mathrm{d}}\right)^{2} \leq r_{\mathrm{d}}^{2}, \\
0 & \text { otherwise, }\end{cases} \\
& \bar{v}(x, y)= \begin{cases}\frac{u_{0}\left(y-y_{\mathrm{d}}\right)}{r_{\mathrm{d}}}+u_{\mathrm{t}} \sin \theta & \text { if }\left(x-x_{\mathrm{d}}\right)^{2}+\left(y-y_{\mathrm{d}}\right)^{2} \leq r_{\mathrm{d}}^{2}, \\
0 & \text { otherwise }\end{cases}
\end{aligned}
$$

As stated previously, the theoretical predictions of the DAF model are to be directly compared to the experimental data taken from $[6,8,11,15]$. The fluid properties for $85 \%$ glycerin, isopropanol, water and hexadecane are shown below in Table 1, taken from the respective experimental studies. For impacts on liquid films, the initial radial velocity $u_{0}=1$, while for impacts on dry surfaces $u_{0}$ is determined from the moving contact line velocity as per the Hoffman-Voinov-Tanner law [8], as follows:

$\theta_{\mathrm{c}}^{3}-\theta_{\mathrm{e}}^{3} \approx c_{\mathrm{T}} u_{0} \frac{\mathrm{We}}{\mathrm{Re}}$,

where $c_{\mathrm{T}} \approx 72 \mathrm{rad}^{3}$ is the experimental constant, initial contact angle $\theta_{\mathrm{c}}=90^{\circ}$, and the static equilibrium contact angle $\theta_{\mathrm{e}}$ is determined from Eq. (18) using experimental data for the radius achieved by the spreading droplet at its equilibrium; both $\theta_{\mathrm{e}}$ and $u_{0}$ are provided in Table 1. Hexadecane impacts are modelled for multiple values of We and Re; however, only one dry surface impact is modelled, which is reflected in the value of $u_{0}$ provided. Both $\theta_{\mathrm{e}}$ and $u_{0}$ are included for only dry surface impacts and reflect the value corresponding to the particular substrate used in each study, which was smooth glass for glycerin and isopropanol and aluminium for hexadecane, while no dry impacts were modelled for water.

The separate conditions and related dimensionless values that were used to fully define the impacts for all simulations are shown in Table 2, again, taken from $[6,8,11,15]$. Oblique impacts were simulated using the same fluid and impact conditions as those modelled by [4], but with no directly comparable experimental data available.

\section{Numerical implementation}

\subsection{Spatial discretisation}

Equations (9)-(12) are solved subject to the attendant boundary conditions, shown in Eq. (14), on a rectangular computational domain $(x, y)=(0, l) \times(0, w)$. This domain consists of regular rectangular cells divided into $n_{\mathrm{x}}$ and $n_{\mathrm{y}}$ cells, respectively, with sides of length $\Delta x=l / n_{\mathrm{x}}$, and width $\Delta y=w / n_{\mathrm{y}}$. Each cell is subdivided using a staggered arrangement of unknowns $\bar{u}, \bar{v}, p$ and $h$, as shown in Fig. 2. Similarly, for axisymmetric flow, the computational domain $r=[0, l]$ is divided into $n_{\mathrm{x}}$ cells with a staggered arrangement for unknowns $\bar{u}, p$ and $h$. For all axisymmetric impacts, the values of $l$ and $w$ were set to $l=w=10$ with the centre of the impact at $\left(x_{\mathrm{d}}, y_{\mathrm{d}}\right)=(0,0)$, for oblique impacts, the domain dimensions were also set to $l=w=10$; however, the centre of impact was set at $\left(x_{\mathrm{d}}, y_{\mathrm{d}}\right)=(-2.5,0)$ to accommodate for increased fluid movement in the $x$-direction. 
Table 2 Fluid dimensionless number values

\begin{tabular}{llcrr}
\hline & $U_{0}(\mathrm{~m} / \mathrm{s})$ & We & Re & Fr \\
\hline Hexadecane [6] & 1.36 & 106 & 618 & 9.7 \\
& 2.17 & 269 & 987 & 15.5 \\
& 3.15 & 566 & 1432 & 22.5 \\
& 3.80 & 824 & 1728 & 27.1 \\
& 4.22 & 1016 & 1919 & 30.1 \\
85\% glycerin [8] & 1.04 & 51 & 27 & 6.7 \\
Isopropanol [11] & 1.17 & 93 & 693 & 8.8 \\
Water [15] & 2.38 & 297 & 9296 & 12.3 \\
\end{tabular}

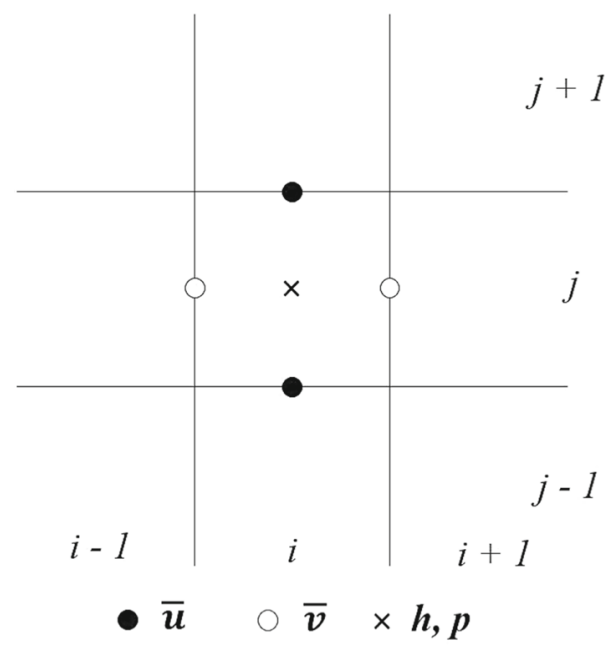

Fig. 2 Staggered mesh method of solution, preventing checkerboard instabilities, for the variables $\bar{u}, \bar{v}, h$ and $p$, denoted by filled circles, open circles and crosses, respectively

The unknown variables required to predict the droplet impact; thickness $h$, pressure $p$ and the velocity components $(\bar{u}, \bar{v})$, are located at cell centres $(i, j)$, and cell faces $(i+1 / 2, j)$ and $(i, j+1 / 2)$, respectively. This staggered mesh arrangement avoids the checkerboard instability which results in a periodic pattern of high and low Pseudo-densities [55]. The instability occurs if central differencing is applied to first-order pressure term derivatives and to the terms in the continuity equation when pressure and velocity components are collocated. Further details concerning the spatial discretisation scheme are available in [38].

\subsection{Temporal discretisation and multigrid solver}

An automatic adaptive time-stepping procedure is utilised to decrease computational resource required, as per [38]. This uses estimates of the local truncation error obtained from the difference between an explicit predictor stage and the current solution stage to optimise the time step size. The local truncation error is subject to a tolerance, $\varepsilon$, which then uses the $\beta$-method to advance the solution in time, with $\beta=0.75$. This value of $\beta$ gives a mix of first and second-order differentiation, giving a balance between efficiency and reliability. Fully explicit second-order equations are used to predict values for the unknowns for both 3D and axisymmetric impacts. The equations are solved using a combination of the Full Approximation Storage (FAS) method and a customised multigrid strategy [47]. The discretised equations are solved using a fixed number of FAS V-cycles on intermediate grid levels with up 
to $10 \mathrm{~V}$-cycles on the first grid level so that residuals are reduced below a specified tolerance. The benefits of using at least one $\mathrm{V}$-cycle at the intermediate level are clear as the relative residuals are reduced by almost two orders of magnitude. Each V-cycle runs through a pre-smoothing, coarse-grid correction and post-smoothing stage. This time-stepping scheme is both efficient and accurate, ensuring small time steps are avoided when the solution varies slowly and enabling the error to be controlled throughout the solution process.

\section{Results and discussion}

\subsection{Axisymmetric impacts}

Axisymmetric impacts on both dry surfaces and liquid films were modelled. Simulations for $85 \%$ glycerin and isopropanol impacting on dry surfaces were compared with [8] and [11], respectively. Water impacting upon a film of depth $h^{*}=0.67$ was also modelled and the numerical results compared with [15]. For full data defining the impacts, refer to Table 2 .

As stated in Sect. 3.2; for each prediction, the time-stepping scheme requires a specific error tolerance, $\varepsilon$, to be defined to adjust the time step according to this value, optimising the time step in a controlled manner. To determine the optimum value of $\varepsilon$, the computational time required to solve different sizes of mesh grid was compared for a variety of values, using a water droplet impact with $\mathrm{We}=297, u_{0}=1$ and $h^{*}=0.67$. Figure 3 shows the relative efficiency of different values of $\varepsilon$ in solving the droplet impacts. Also included in the figure is the total computational time without an adaptive time-stepping scheme. From the figure, it can be seen that both $\varepsilon=0.1$ and $\varepsilon=1$ are the most efficient values for different values of $n_{\mathrm{x}}$. As discussed below, all droplet impact simulations were solved using a grid of $n_{\mathrm{x}} \geq 2048$; therefore, for all values of $n_{\mathrm{x}}$ used, $\varepsilon=0.1$ provided the most efficient solution. For the simulations using the adaptive time-stepping scheme, this improved the computational time required by $93.8 \%$ for $n_{\mathrm{x}}=2048,93.1 \%$ for $n_{\mathrm{x}}=4096$ and $92.8 \%$ for $n_{\mathrm{x}}=8192$, in comparison with a non-adaptive time step.

To ensure the accuracy of results, grid independency studies were carried out to ensure the computational domain was divided into enough cells such that the results were independent of both $n_{\mathrm{x}}$ and $n_{\mathrm{y}}$. Two studies were undertaken for both dry and wet surfaces, with the assumption that dry surfaces would require a finer grid due to the length scale of the precursor films compared with liquid films. Both studies were $2 \mathrm{D}$ axisymmetric models, and as all 3D models used $n_{\mathrm{x}}=n_{\mathrm{y}}$, the results are valid across both 2D and 3D simulations.

Figure 4 shows the results of the grid independency study for liquid films, conducted for a water droplet impact with We $=297, u_{0}=1, h^{*}=0.67$ and a time-shift of $t_{\mathrm{S}}=0.35$, using both crown height and diameter to determine at what number of cells the result was independent. From the studies, for height and diameter, between $n_{\mathrm{x}}=2048$ and $n_{\mathrm{x}}=4096$, there is a $4.5 \%$ height change and $0.2 \%$ diameter change in the final values; therefore, $n_{\mathrm{x}}=2048$ is considered to be sufficient to ensure accurate results within these error ranges.

In addition to the grid independency study, the figure also compares both the evolution of crown diameter (a) and height (b), as defined in Fig. 1b, over time against experimental data from [15], highlighting the difference in accuracy of the DAF predicting horizontal and vertical action in the droplet impact. In (a) the numerical prediction of crown diameter evolution is almost identical to the experimental data from [15], which is found to have the relation $d=C \cdot\left(t-t_{0}\right)^{n}$ where for this case; $C=2.06, n=0.45$ and $t_{0}=0$. In (b) DAF predicts the crown to form and breakdown much faster than experimental data, as well as underestimating the maximum crown height. This could be due to two related reasons; foremost of which is that vertical inertia is not taken into account, so there is no momentum to carry the fluid to the correct height. This is due to all second order, $\mathscr{O}\left(\mathscr{E}^{2}\right)$, or higher, terms in the DAF equations being factored out, meaning the vertical DAF equation only consists of gravity, surface tension and pressure forces as seen in Eq. (12). As horizontal inertia is considered within the DAF equations, the diameter shows strong agreement. A further reason for incorrect predictions of the crown height is the initial droplet and velocity profile used in the model. From Fig. 2 in [11], it can be seen that when the droplet impacts and reaches $h=0.6300$, the fluid is not a perfect spherical cap and there is already spreading of the droplet. Though [11] shows this for a dry surface impact, for a liquid film impact, this will cause the fluid outside and below the impact site to have been 


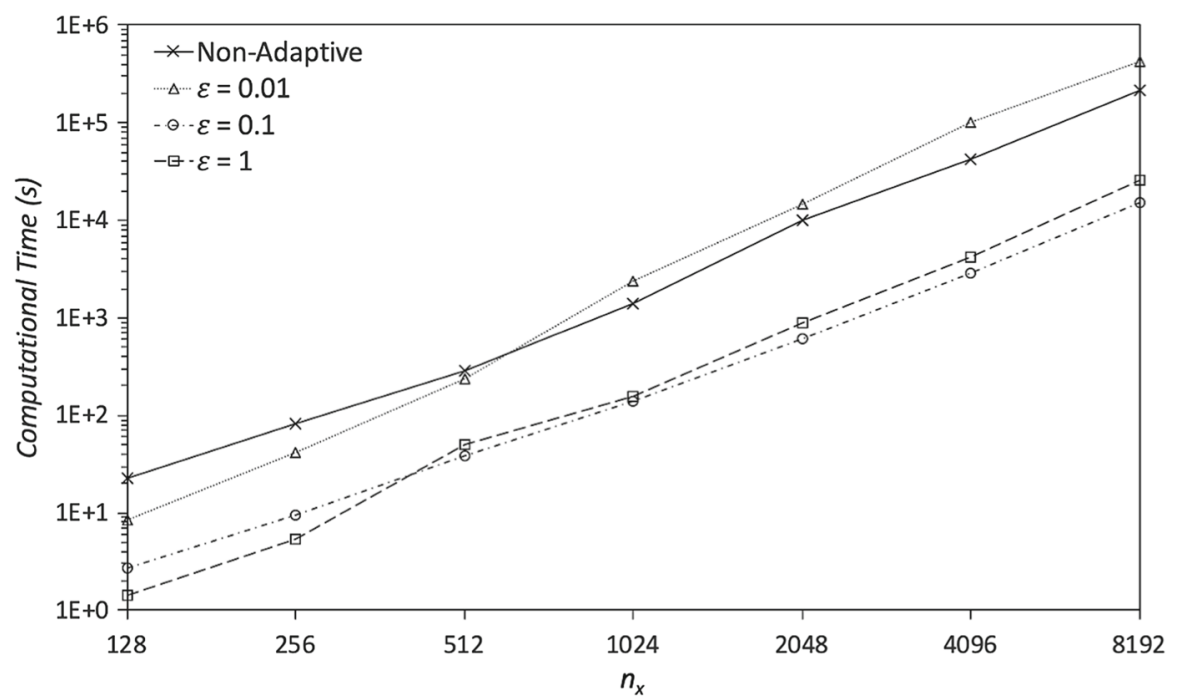

Fig. 3 Computational time dependence on number of cells $n_{\mathrm{x}}$, taking a water droplet impacting upon a liquid film as a benchmark, for the indicated values of local truncation error tolerance $\varepsilon$, where $\mathrm{We}=297, u_{0}=1$ and $h^{*}=0.67$. Computations are conducted on the Linux-based Hamilton cluster environment
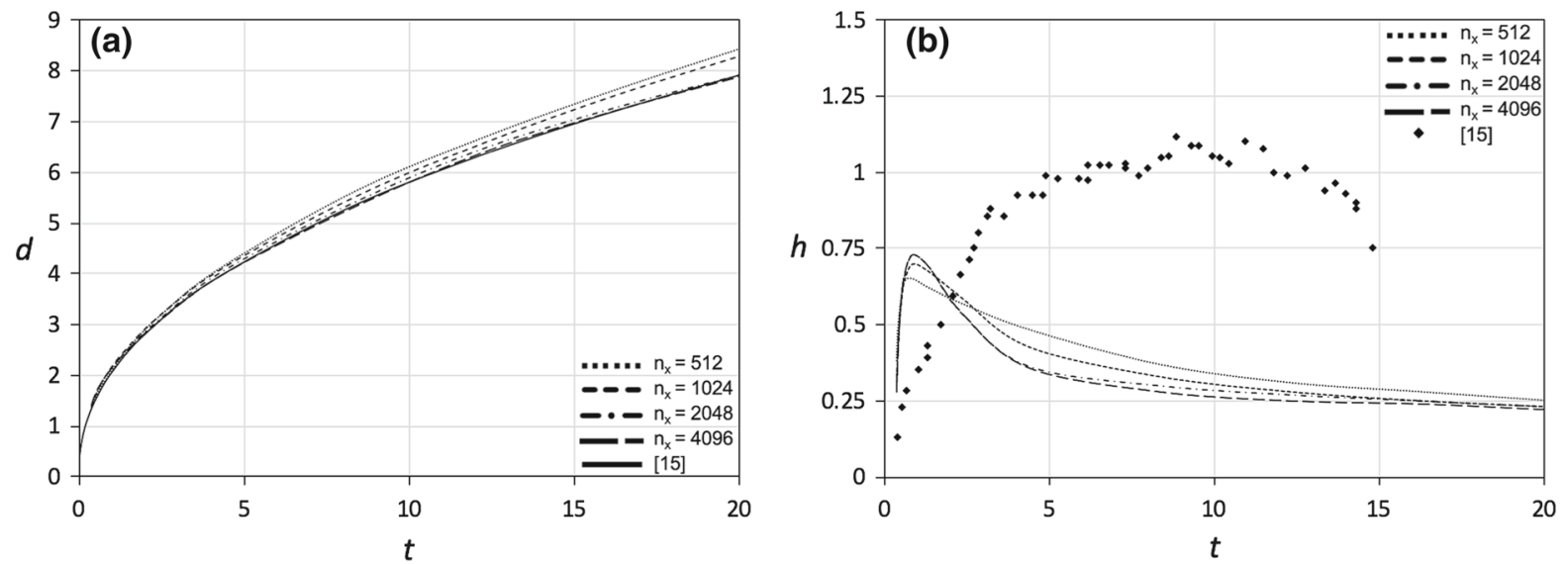

Fig. 4 Diameter and height of the crown formed for a water droplet impacting upon a liquid film: grid independency study and comparison with the complementary experimental data [15], for; a crown diameter, b crown height, where We $=297, u_{0}=1$, $h^{*}=0.67$ and applying a time-shift of $t_{\mathrm{s}}=0.35$, as per Fig. 3

disturbed before the point at which the DAF prediction begins. This forces the fluid down further, generating more vertical momentum than the spherical cap predicts and therefore taking longer to form and dissipate the crown.

Figure 5 shows the results of the dry surface grid independency study, carried out for a glycerin impact with We $=51, \theta_{\mathrm{e}}=63.8^{\circ}$, according to the relation in Eq. (17), $u_{0}=0.02$ and a precursor film thickness of $h^{*}=0.001$. Though two values of precursor film thickness are compared in the discussion below, the fact that $h^{*}=0.001$ is a smaller scale than $h^{*}=0.01$, allows the results of the grid independency study to be applied to both precursor films. From the figure, it can be seen that between $n_{\mathrm{x}}=8192$ and $n_{\mathrm{x}}=16384$ there is only $1.1 \%$ change in the final spreading diameter; therefore, $n_{\mathrm{x}}=8192$ was used for all dry surface impact models.

Figure 6 shows the diameter of the same glycerin impact over time where $\mathrm{We}=51, \theta_{\mathrm{e}}=63.8^{\circ}$ and $u_{0}=0.02$, but compares two values of precursor film, $h^{*}=0.01$ and $h^{*}=0.001$, with each other and experimental data from [8]. A time-shift of $t_{\mathrm{s}}=0.47$ is required to align the initial condition of the DAF impact where $h_{\mathrm{d}}=r_{\mathrm{d}}=0.6300$ with the corresponding instant of the experimental data. A precursor film is required for dry impacts due to the nature 


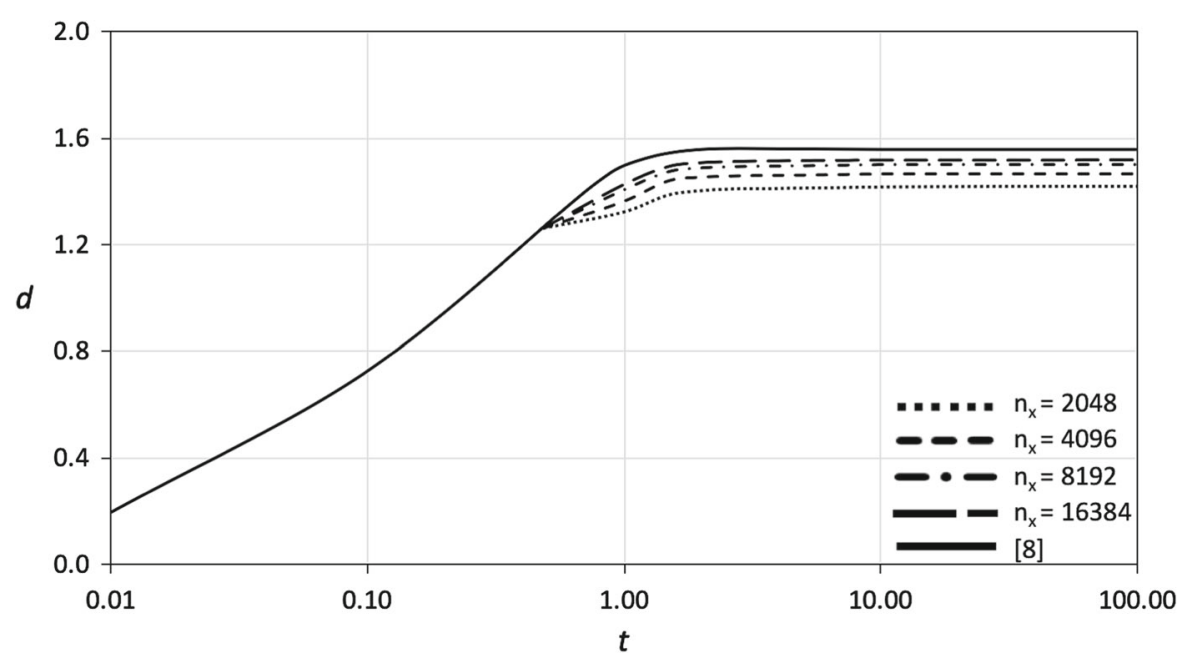

Fig. 5 Spreading diameter of a glycerin droplet impact upon a dry surface vs. time: grid independency study and comparison with the complementary experimental data [8], for $\mathrm{We}=51, \theta_{\mathrm{e}}=63.8^{\circ}, u_{0}=0.02, h^{*}=0.001$ and applying a time-shift of $t_{\mathrm{s}}=0.47$

of DAF, which requires a depth of fluid at every point in the computational domain to be able to be solved. From the figure it can be seen that decreasing the thickness of $h^{*}$ by a factor of 10 results in a more accurate prediction, with $d=1.499$ at $t=100$ for $h^{*}=0.001$ and $d=1.467$ for $h^{*}=0.01$. This is compared with $d=1.560$ from the experimental data and shows an increase in accuracy of $2.1 \%$ for the lower value of $h^{*}$. As the thicker precursor film is closer in scale to the droplet thickness $h$, it will have a greater effect upon the spreading rate and diameter, increasing the error from the experimental data. The reason for the underprediction of both film thicknesses is that until total equilibrium the droplet may not be a perfect spherical cap and may have a slight rim, as seen in Fig. 1a in [8]. This would lead to an overprediction of the static equilibrium contact angle; however, once the droplet reached complete equilibrium, the prediction should match the experimental data for both precursor films. The agreement of the prediction for $h^{*}=0.001$ is strong, with the diameter at $t=100$ within $4.5 \%$ of the experimental data.

Figure 7 shows the diameter of an isopropanol impact over time with $\mathrm{We}=93, \theta_{\mathrm{e}}=6.5^{\circ}$ and $u_{0}=0.40$. As with the glycerin impact, two values of precursor film are compared with the experimental data, with a time-shift of $t_{\mathrm{s}}=0.21$. The results of this simulation show less agreement with experimental data than glycerin. As isopropanol has a much lower viscosity and lower static equilibrium contact angle it takes a lot longer to reach equilibrium. At $t=10, h^{*}=0.001$ is $4.2 \%$ above the experimental data and $h^{*}=0.01$ is $7.1 \%$ above, giving an increase in accuracy of the prediction by $3.0 \%$. This discrepancy can again be explained by the physical droplet not being a perfect spherical cap at both the initial impact and at equilibrium. As the initial modelled droplet starts at a lower height than the actual impact, there is less vertical acceleration, reducing the spreading rate. The experimental data from [11] also did not reach times past $t=8$, so though a line of best fit was used to predict the droplet diameter at equilibrium, it is possible the spreading rate could have changed beyond this time, and would otherwise align more with the DAF predictions. Decreasing $h^{*}$ results in more computational resource being required; however, for this 2D axisymmetric case, this is not a limiting factor. In contrast, for 3D cases, the simulation takes much longer and a compromise between accuracy and time has to be considered.

\subsection{D normal impacts}

3D normal impacts on both dry surfaces and liquid films were modelled and compared with the photographic compendium from [6], recorded at the frame rate of 2,000 frames/s; the most viscous fluid hexadecane was considered. The same adaptive time-stepping scheme was used, as per Fig. 3, with $\varepsilon=0.1$. A grid size of $n_{\mathrm{x}} \times n_{\mathrm{y}}=2048 \times 2048$ was used for 3D impacts. This size of mesh is not grid independent for dry surface impacts, as shown in Fig. 5; 


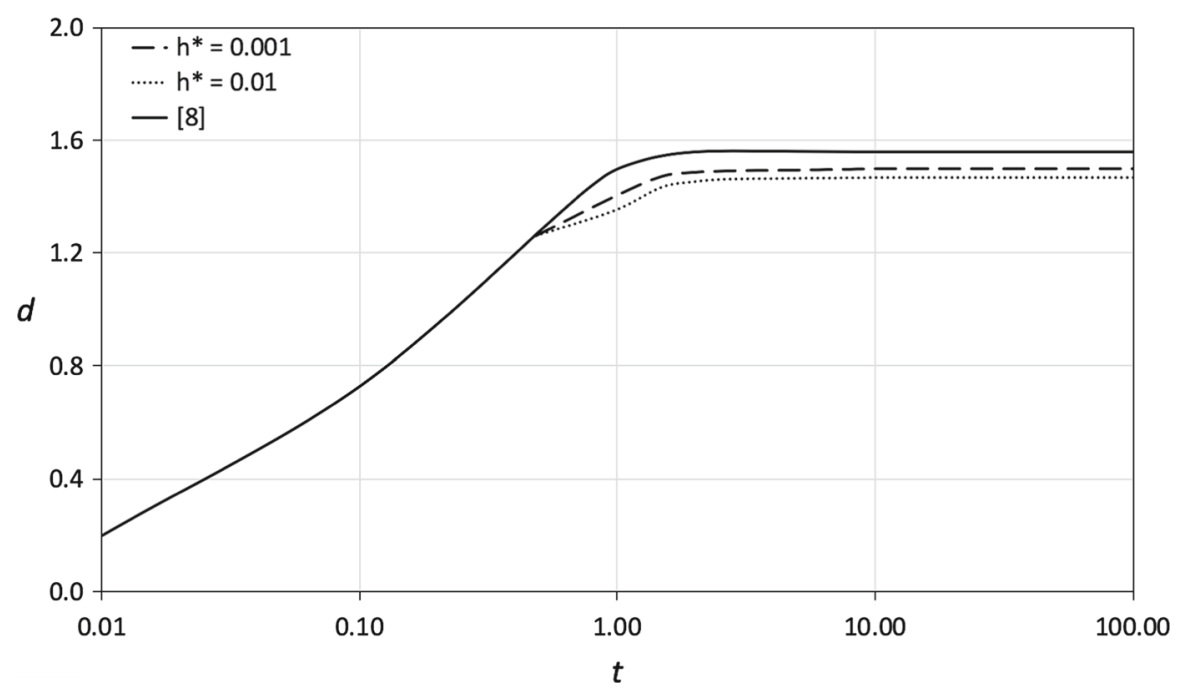

Fig. 6 Spreading diameter of a glycerin droplet impacting upon a dry surface vs. time: effect of precursor film thickness $h^{*}$ and comparison with the complementary experimental data [8], for We $=51, \theta_{\mathrm{e}}=63.8^{\circ}, u_{0}=0.02$ and applying a time-shift of $t_{\mathrm{s}}=0.47$

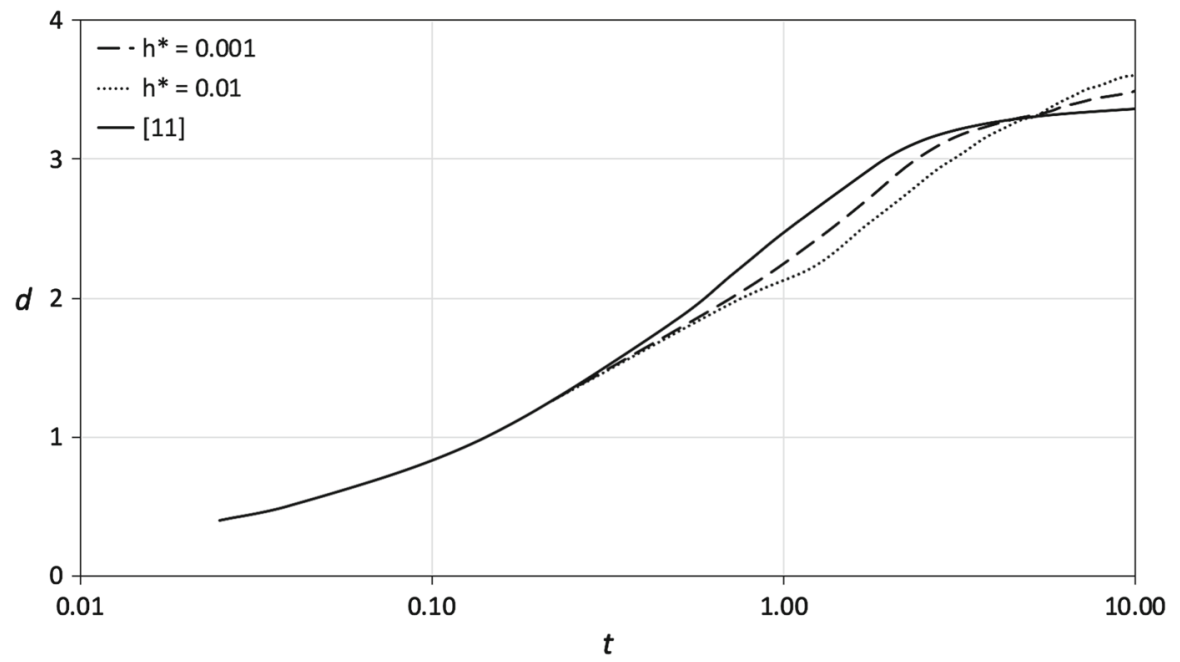

Fig. 7 Spreading diameter of a isopropanol droplet impacting upon a dry surface vs. time: effect of precursor film thickness $h^{*}$ and comparison with the complementary experimental data [11], for We $=93, \theta_{\mathrm{e}}=6.5^{\circ}, u_{0}=0.40$ and applying a time-shift of $t_{\mathrm{s}}=0.21$

however, due to the time constraints, performing a simulation with $n_{\mathrm{x}} \times n_{\mathrm{y}}=4096 \times 4096$ or $n_{\mathrm{x}} \times n_{\mathrm{y}}=8192 \times 8192$ was infeasible. Any $3 \mathrm{D}$ results for dry surface impacts therefore have a potential $\pm 5.2 \%$ error range. Each $3 \mathrm{D}$ DAF prediction corresponds to the same time as the frame shown in the adjacent experimental figure and is viewed from a high angle. For full data defining the impacts, refer to Table 2.

The dry surface hexadecane impacts were solved in 3D to compare with [6] and provided qualitative confirmation of the analysis of the glycerin and isopropanol impacts. Figure 8 shows a 3D hexadecane droplet impacting on a dry surface for $\mathrm{We}=106, \theta_{\mathrm{e}}=11.2^{\circ}$ and $u_{0}=0.31$, with no time-shift. $\theta_{\mathrm{e}}$ was again calculated using the relation in Eq. (18), having assumed Frame 12 in Fig. 8 a shows the equilibrium state and measuring $d=3$ in this frame. No time-shift was required for this impact as the initial droplet shown in Frame 1 mirrors the prescribed spherical cap initial condition. A precursor film of $h^{*}=0.01$ was used to reduce computational time needed for the calculation. The figure shows strong agreement with the experimental data, showing similar spreading rates and diameters throughout the impact, reaffirming the results found in Sect. 4.1. The depth of the droplet across the diameter is 


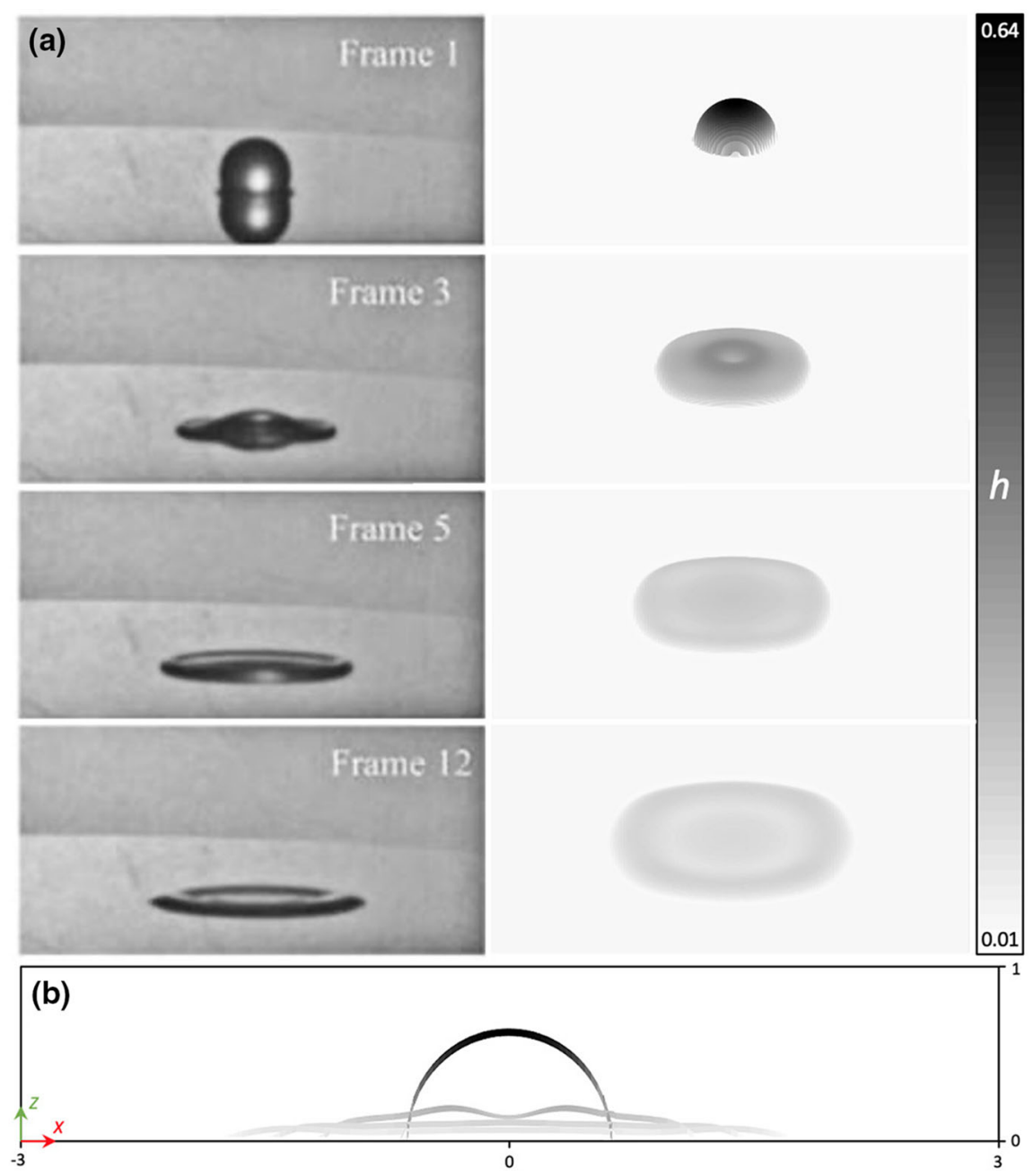

Fig. 8 Hexadecane droplet impinging upon a dry surface: comparison of DAF prediction with the complementary experimental data, Fig. 2 from [6], for $\mathrm{We}=106, \theta_{\mathrm{e}}=11.2^{\circ}, u_{0}=0.31, h^{*}=0.01$ and applying no time-shift $t_{\mathrm{s}}=0$ for times $t=0.00,0.68,1.36$ and 3.74: a free-surface profiles, $\mathbf{b}$ cross-section profiles through the centre of the impact

also accurate in the numerical prediction. In Frame 3, the residual shape of the initial impact is visible, which is reflected in the DAF prediction, in Frame 12, an outer rim is formed which is thicker than the internal diameter. The accuracy of the droplet height prediction in this figure is due to minimal vertical inertia in a dry surface impact compared to a liquid film impact. As there is little vertical movement after impact, the consideration of gravitational and pressure forces are sufficient to describe the droplet height.

Figures 9 and 10 show 3D hexadecane impacts on thin films, where $h^{*}=0.1$, for $\mathrm{We}=269$ and $\mathrm{We}=566$, respectively, with $u_{0}=1$. Figure 9 has a time-shift of $t_{\mathrm{s}}=0.51$ and Fig. 10 a time-shift of $t_{\mathrm{s}}=0.17$. For the same thickness of film, faster velocities require smaller time-shifts due to the time required to reach the condition of $h_{\mathrm{d}}=r_{\mathrm{d}}=0.6300$. As with water impacts, the crown diameter is predicted accurately in both cases. The thickness of the crown, however, as predicted by DAF is larger than the experimental data, no rim is shown in the predictions and the crown height is again underestimated. The inaccuracies of the predictions of both crown thickness and height are both due to the lack of vertical inertia in the model. The kinematic discontinuity of the crown is not as prominent as in the physical situation, so more fluid forms the crown due to surface tension and viscosity, described by Re and We, which also explains the lack of a rim. However, a rim would be unable to be predicted by DAF 


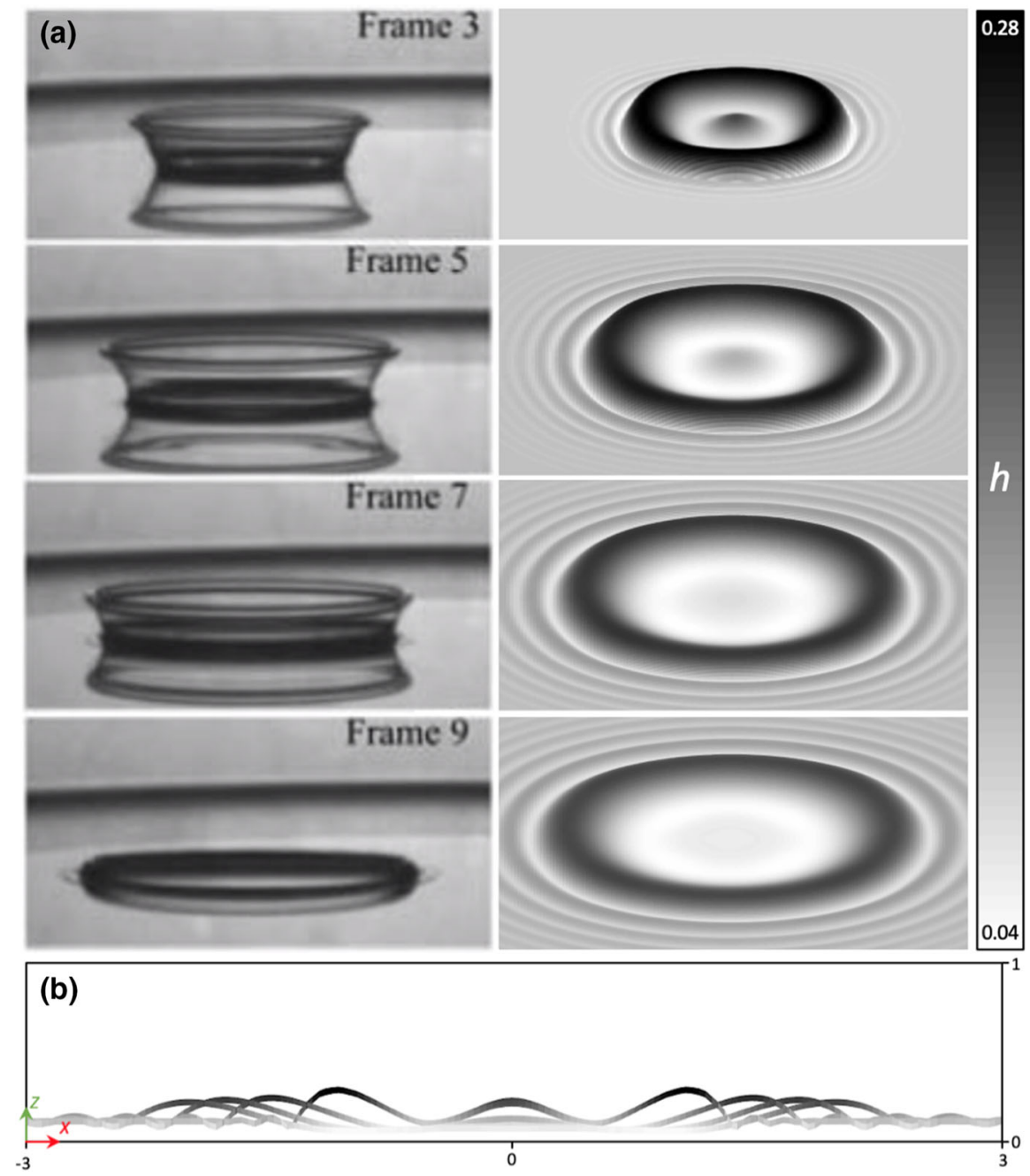

Fig. 9 Hexadecane droplet impinging upon a thin liquid film: comparison of DAF prediction with the complementary experimental data, Fig. 4 from [6], for $\mathrm{We}=269, u_{0}=1, h^{*}=0.1$ and applying a time-shift of $t_{\mathrm{s}}=0.51$ for times $t=1.12,2.20,3.29$ and 4.37: a free-surface profiles, $\mathbf{b}$ cross-section profiles through the centre of the impact

anyway due to the multivalued free surface. In both figures the DAF prediction also shows capillary waves being produced, which only begin to form in later frames in the physical situations. In the experimental data, the capillary waves are only produced as the crown falls, and again due to the lack of vertical inertia in the DAF predictions, the crown begins to fall for the DAF predictions quicker than the physical situation, leading to capillary waves being produced earlier.

Figure 10 also shows splash occurring after impact, between Frames 7 and 13, with the DAF method diverging within this timeframe. Though Fig. 10 shows some slight instabilities in the crown, Fig. 11 shows the point at which the method diverges, corresponding to approximately Frame 10, for the same impact but with a grid size of $n_{\mathrm{x}} \times n_{\mathrm{y}}=512 \times 512$. This figure highlights the creation of inertia-driven instability within the crown and how this causes points on the crown to become too thin for the mesh grid used. The coarser mesh better shows the instabilities due to the resolution of the figures produced, the instabilities are still present for a grid of $n_{\mathrm{x}} \times n_{\mathrm{y}}=2048 \times 2048$; however, this resolution makes it more difficult to observe the exact gridpoints the instabilities cause the DAF method to diverge. 


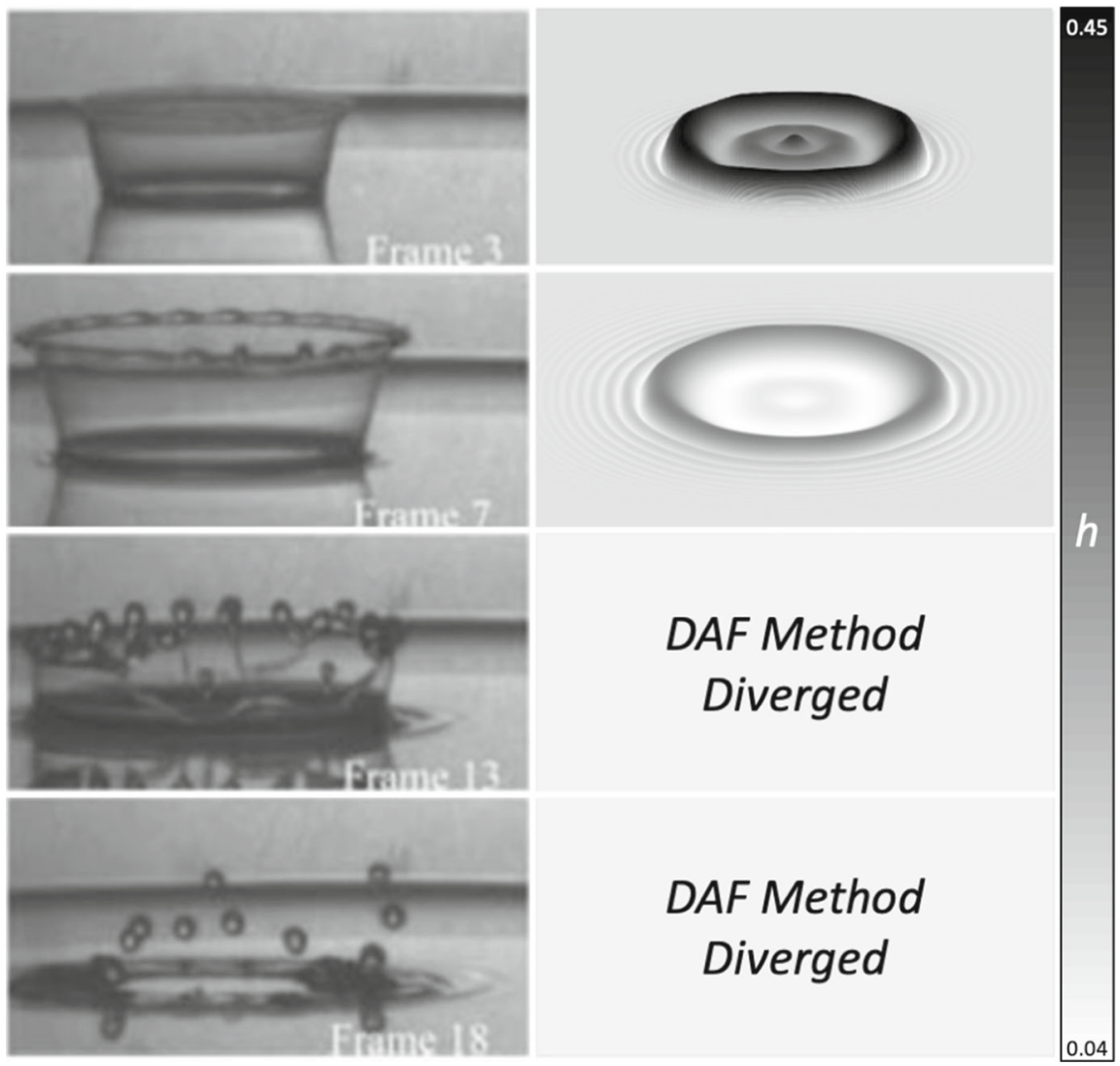

Fig. 10 Hexadecane droplet impinging upon a thin liquid film: comparison of DAF prediction with the complementary experimental data, Fig. 9 from [6], for We $=566, u_{0}=1, h^{*}=0.1$ and applying a time-shift of $t_{\mathrm{s}}=0.17$ for times $t=0.62,5.34,10.07$ and 14.07

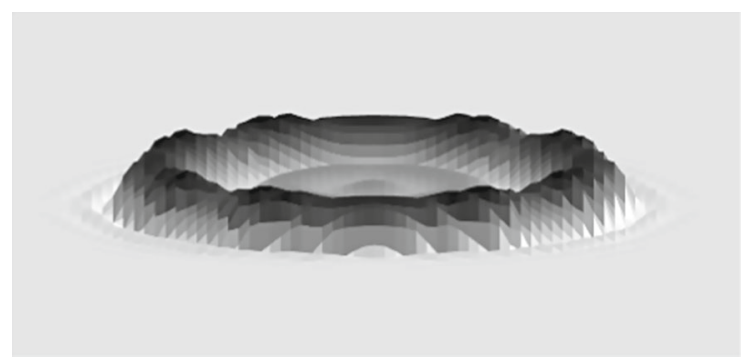

Fig. 11 Instabilities over the crown formed for the hexadecane droplet impact considered in Fig. 10 for a grid size $n_{\mathrm{x}}=n_{\mathrm{y}}=512$ and corresponding to the same time as Frame 10

Without knowing the exact time at which splash occurred in the physical impact, to further investigate whether splash is predicted correctly by DAF, different cases were explored. Tables 3 and 4 compare the occurrence of splash in experimental data with divergence of DAF. From the tables, it can be seen that for all but one case, the method diverges when splash occurs. For all these cases, the divergence occurred within the correct timeframe when compared to [6]. The only case in which the method diverging and presence of splash in the experimental data do not correspond is where the splash was minimal. In this case, it may be that the precursor film of $h^{*}=0.01$ prevents the splashing due to surface tension or by being of a similar scale, and the number of cells in the computational domain would need to be increased to detect the splashing. By observation, not all splashes in these cases occur in the same manner as in Fig. 11, as some happen instantaneously with no crown formed. For all cases, it is assumed 
Table 3 Splash in Experimental Observations

\begin{tabular}{lllll}
\hline We & $h^{*}$ & & & \\
\cline { 2 - 5 } & 0.0 & 0.1 & 1.0 & 10.0 \\
\hline 106 & No & No & No & No \\
269 & Yes & No & No & No \\
566 & Yes & Yes & No & No \\
824 & Yes & Yes & Yes & Yes \\
1016 & Yes & Yes & Yes & Yes \\
\hline
\end{tabular}

Table 4 Divergence of DAF due to Splash in DAF Predictions

\begin{tabular}{lllll}
\hline We & $h^{*}$ & & & \\
\cline { 2 - 5 } & 0.0 & 0.1 & 1.0 & 10.0 \\
\hline 106 & No & No & No & No \\
269 & No & No & No & No \\
566 & Yes & Yes & No & No \\
824 & Yes & Yes & Yes & Yes \\
1016 & Yes & Yes & & Yes \\
\hline
\end{tabular}

that the reasons the DAF method diverges and therefore the prediction of splash is based upon the fluid free surface become multivalued or too thin for the mesh to solve.

\subsection{D oblique impacts}

3D oblique impacts on liquid films were modelled. As no experimental data were available to compare the impacts with, attempts to compare the model with the analytical solution from [4] were made. For oblique impacts, a finer grid was required of $n_{\mathrm{x}} \times n_{\mathrm{y}}=4096 \times 4096$ to obtain a solution. This finer grid increased the time taken for the simulations to run; therefore, only small time scales could be predicted. The results in [4] are shown for $t=5,10$ and 15, but due to the limitation of computational resource, no comparison could be made for $t=15$.

The predicted crown shape for an oblique water impact with We $=297, h^{*}=0.67$, impact angle $\theta=45^{\circ}$ and $u_{0}=u_{\mathrm{t}}=1$ for times $t=0.1,0.3,1$ and 3 is shown in Fig. 12a-d. The figure shows the droplet moving laterally, with most action between $t=0.3$ and $t=1$, with the diameter of the impact doubling and crown height increasing. There could be the same issue with predicting crown height as with earlier simulations, in that the lack of consideration of vertical inertia predicts most movement at an earlier time than the physical reality. This could also mean that the crown is under-predicted and the actual impact would show more dramatic dynamics. At each time, instabilities within the crown can be seen, which could be a mechanism of splashing, as in Sect. 4.2. However, these instabilities smooth out and the solution does not diverge, possibly due to the larger grid size used.

Figure 12e shows a comparison of the analytical solution presented of the shape of the external base of the crown for $t=5$ and $t=10$ with the predictions in Fig. 7b from [4]. The figure shows [4] to predict greater crown diameter than that of DAF at these times. As shown in Fig. 3 DAF predicts the crown diameter for normal impacts well, while it is expected that the analytical solution [4] overpredicts the crown diameter since it is obtained ignoring gravity, viscosity and surface tension, i.e. in the limits of $\mathrm{We} \rightarrow \infty, \mathrm{Re} \rightarrow \infty$ and $\mathrm{Fr} \rightarrow \infty$. Data from [15] also demonstrate that the crown diameter increases with impact velocity as well as We, Re and Fr. 

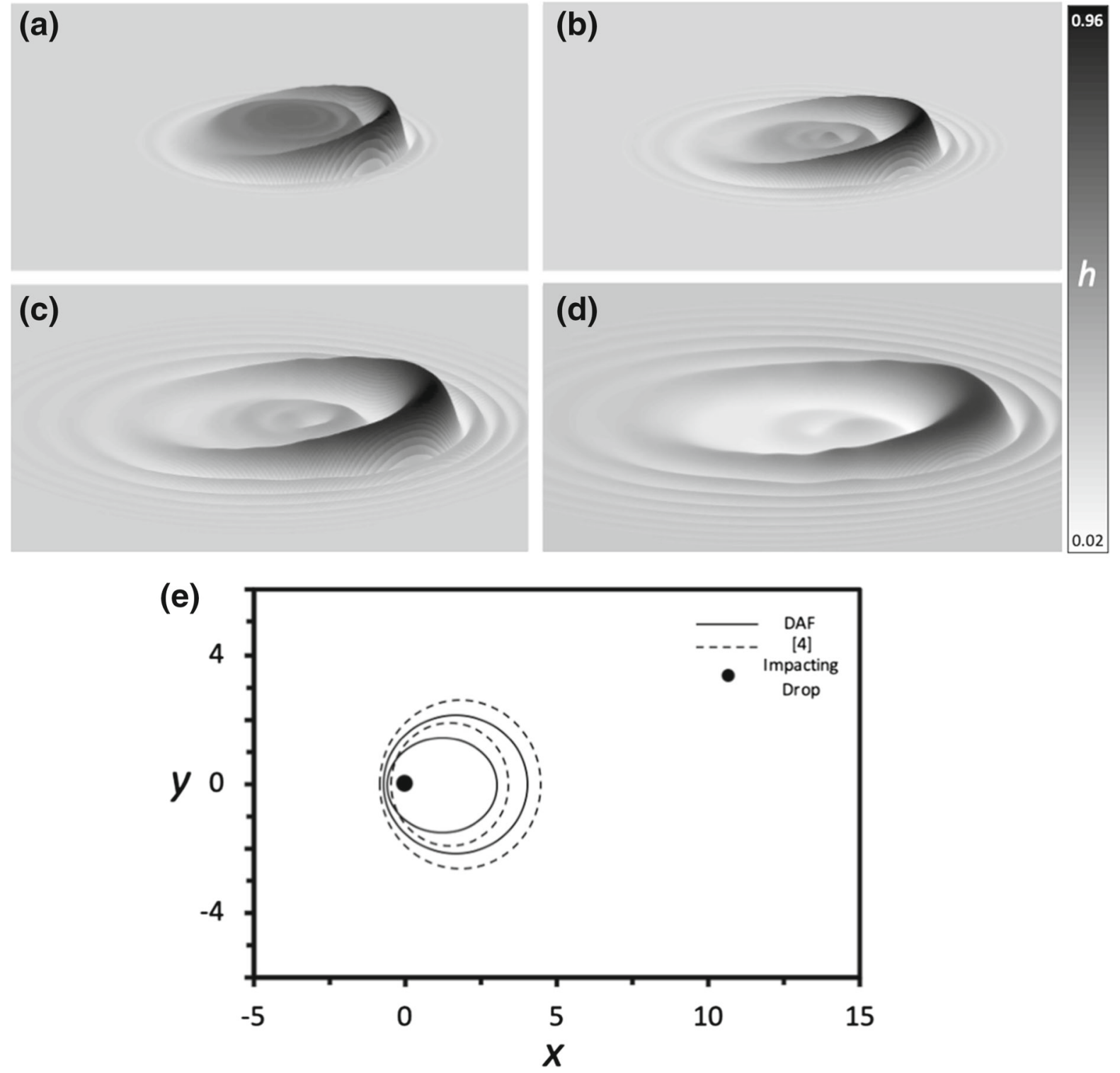

Fig. 12 Oblique impact $\left(\theta=45^{\circ}\right)$ of a water droplet upon a liquid film: DAF prediction for We $=297, u_{0}=u_{\mathrm{t}}=1$ and $h^{*}=0.29$; $\mathbf{a} t=0.1, \mathbf{b} t=0.3, \mathbf{c} t=1, \mathbf{d} t=3$, e comparison of DAF prediction for shape of the external base of the crown with analytical solution presented in Fig. 7b from [4] for time instants $t=5$ and 10

\section{Conclusions}

This study used the DAF method to predict a range of droplet impacts, for different fluids, film thicknesses and impact angles, comparing the predictions to experimental data to validate the accuracy of DAF in droplet impact modelling. For dry surface impacts, there is strong agreement between the theoretical prediction and experimental data. Wet surface impacts contrasted the accuracy of the DAF in predicting horizontal and vertical movement in droplet impacts. While predictions of droplet and crown diameters were accurate, crowns were predicted as developing and breaking down too quickly, with overall height underestimated. This is due to the lack of consideration of vertical 
inertia in the DAF model used, caused by ignoring all terms of order $\mathscr{O}\left(\mathscr{E}^{2}\right)$ or higher in the DAF equations. The vertical DAF equation therefore only consists of gravity and pressure terms.

In all but one case, DAF also predicted splash within the correct time range, by the fact the method diverges. In the exceptional case, there was minimal splashing and therefore to make DAF a greater predictor of splash, a finer mesh is required. The splash shown in Fig. 11 occurred due to instabilities within the crown; however, different types of splash occurred for different cases, all of which are assumed to cause instabilities where the fluid becomes too thin for the mesh to solve, or the free surface became multivalued.

To progress the work, further adjustments need to be made to the model. Foremost, the long-wave assumption used in the DAF predictions will have to be altered so that vertical inertia is incorporated into the model and crown height can be modelled as accurately as the diameter. To better predict splash, finer meshes will be required, though this increases the computational resource required for each simulation. Therefore, further optimisation of the model is required. In its current state, this model can therefore accurately predict spreading and splashing, which are married in terms of applicability. The DAF prediction could also be used to investigate cases in which spray density is high enough that the assumption that behaviour of a single drop represents that of a spray no longer applies. Further work could therefore investigate how multiple droplets interact when impacting both near to or on top of each other. The model could be particularly useful in fields where thin-film deposition is of importance, such as spray painting, surface cleaning and ink-jet printing.

Open Access This article is licensed under a Creative Commons Attribution 4.0 International License, which permits use, sharing, adaptation, distribution and reproduction in any medium or format, as long as you give appropriate credit to the original author(s) and the source, provide a link to the Creative Commons licence, and indicate if changes were made. The images or other third party material in this article are included in the article's Creative Commons licence, unless indicated otherwise in a credit line to the material. If material is not included in the article's Creative Commons licence and your intended use is not permitted by statutory regulation or exceeds the permitted use, you will need to obtain permission directly from the copyright holder. To view a copy of this licence, visit http://creativecommons.org/licenses/by/4.0/.

\section{Appendix A. DAF derivation}

As discussed in Sect. 2.1, solving Eqs. (3) and (4) to the boundary conditions (5)-(8), although possible, is computationally challenging and would require a lot of computer resource and time in order to obtain a mesh-independent solution. To bypass this it is possible to use the long-wave approximation based on the assumption that the ratio $\mathscr{E}=H_{0} / L_{0} \ll 1$, showing that the characteristic length scale in the vertical direction $H_{0}$ is much smaller than the characteristic length scale in the horizontal direction $L_{0}$. In addition to aiding the theoretical predictions of the splash, this assumption effectively reduces the dimensionality of the problem by one. Formulating (3) and (4) in terms of scales $H_{0}$ and $L_{0}$ leads to:

$$
\begin{aligned}
& \mathscr{E} \operatorname{Re}\left[\frac{\partial u}{\partial t}+u \frac{\partial u}{\partial x}+v \frac{\partial u}{\partial y}+w \frac{\partial u}{\partial z}\right]=-\frac{\operatorname{Re}}{\mathrm{We}} \frac{\partial p}{\partial x}+\mathscr{E}^{2}\left(\frac{\partial^{2} u}{\partial x^{2}}+\frac{\partial^{2} u}{\partial y^{2}}\right)+\frac{\partial^{2} u}{\partial z^{2}}, \\
& \mathscr{E} \operatorname{Re}\left[\frac{\partial v}{\partial t}+u \frac{\partial v}{\partial x}+v \frac{\partial v}{\partial y}+w \frac{\partial v}{\partial z}\right]=-\frac{\operatorname{Re}}{\mathrm{We}} \frac{\partial p}{\partial y}+\mathscr{E}^{2}\left(\frac{\partial^{2} v}{\partial x^{2}}+\frac{\partial^{2} v}{\partial y^{2}}\right)+\frac{\partial^{2} v}{\partial z^{2}}, \\
& \mathscr{E}^{3} \operatorname{Re}\left[\frac{\partial w}{\partial t}+u \frac{\partial w}{\partial x}+v \frac{\partial w}{\partial y}+w \frac{\partial w}{\partial z}\right]=-\frac{\operatorname{Re}}{\mathrm{We}} \frac{\partial p}{\partial z}+\mathscr{E}^{4}\left(\frac{\partial^{2} w}{\partial x^{2}}+\frac{\partial^{2} w}{\partial y^{2}}\right)+\mathscr{E}^{2} \frac{\partial^{2} w}{\partial z^{2}}-\frac{\operatorname{Re}}{\mathrm{Fr}^{2}}, \\
& \frac{\partial u}{\partial x}+\frac{\partial v}{\partial y}+\frac{\partial w}{\partial z}=0 .
\end{aligned}
$$

The boundary conditions (7) and (8) become:

$$
-\left.p\right|_{z=h}+\left.\left\{\frac{2 \mathscr{E}^{2}\left(-\frac{\partial u}{\partial z} \frac{\partial h}{\partial x}-\frac{\partial v}{\partial z} \frac{\partial h}{\partial y}+\frac{\partial w}{\partial z}\right)+\mathscr{O}\left(\mathscr{E}^{4}\right)}{1+\mathscr{E}^{2}\left[\left(\frac{\partial h}{\partial x}\right)^{2}+\left(\frac{\partial h}{\partial y}^{2}\right)\right]}\right\}\right|_{z=h}=\mathscr{E}^{3} \frac{\operatorname{Re}}{\mathrm{We}} \nabla \frac{\nabla h}{\sqrt{1+\mathscr{E}^{2}\left[\left(\frac{\partial h}{\partial x}\right)^{2}+\left(\frac{\partial^{2}{ }^{2}}{\partial y}\right)\right]}},
$$




$$
\begin{aligned}
\left(\alpha_{\mathrm{t}} \frac{\partial u}{\partial z}+\right. & \left.\beta_{\mathrm{t}} \frac{\partial v}{\partial z}\right)\left.\right|_{z=h}+\mathscr{E}^{2}\left\{\left(\alpha_{\mathrm{t}} \frac{\partial h}{\partial x}+\beta_{\mathrm{t}} \frac{\partial h}{\partial y}\right)\left(-\frac{\partial u}{\partial z} \frac{\partial h}{\partial x}-\frac{\partial v}{\partial z} \frac{\partial h}{\partial y}+2 \frac{\partial w}{\partial z}\right)\right. \\
& \left.+\alpha_{\mathrm{t}}\left[-2 \frac{\partial u}{\partial x} \frac{\partial h}{\partial x}-\left(\frac{\partial u}{\partial y}+\frac{\partial v}{\partial x}\right) \frac{\partial h}{\partial y}+\frac{\partial w}{\partial x}\right]+\beta_{\mathrm{t}}\left[-2 \frac{\partial v}{\partial y} \frac{\partial h}{\partial y}-\left(\frac{\partial u}{\partial y}+\frac{\partial v}{\partial x}\right) \frac{\partial h}{\partial x}+\frac{\partial w}{\partial y}\right]\right\}\left.\right|_{z=h}+\mathscr{O}\left(\mathscr{E}^{4}\right)=0
\end{aligned}
$$

By neglecting all terms of $\mathscr{O}\left(\mathscr{E}^{2}\right)$ or higher, the system simplifies considerably and can be averaged over the depth of the fluid. Equation (24) therefore reduces to:

$\frac{\partial p}{\partial z}=-\frac{\mathrm{We}}{\mathrm{Fr}^{2}} g$.

Integrating Eq. (28) with respect to $z$ and applying boundary condition (7), along with the disjoining pressure $\Pi(h)$, in Eq. (13), that is introduced to alleviate the singularity that would otherwise occur at the droplet's dynamic contact line, gives Eq. (12).

Depth-averaging the continuity equation (25) using Leibniz's rule and applying boundary conditions (5) and (6), leads to the depth-averaged equation (11) for the conservation of mass:

$$
\int_{0}^{h}\left(\frac{\partial u}{\partial x}+\frac{\partial v}{\partial y}+\frac{\partial w}{\partial z}\right) \mathrm{d} z=\frac{\partial h}{\partial t}+\frac{\partial}{\partial x}(h \bar{u})+\frac{\partial}{\partial y}(h \bar{v})=0,
$$

where the depth-averaged components of velocity are

$\bar{u}=\frac{1}{h} \int_{0}^{h} u \mathrm{~d} z, \bar{v}=\frac{1}{h} \int_{0}^{h} v \mathrm{~d} z$.

The DAF of the momentum equations (22) and (23) is obtained in three stages: first the pressure gradient, then the diffusion terms and finally the advection terms are averaged. In the case of the $u$-momentum equation (24), making use of boundary conditions (5) and (6) and Eqs. (26) and (27), while noting that the average of fluctuations about the average are zero, gives, in order:

$$
\begin{aligned}
& \int_{0}^{h} \frac{\partial p}{\partial x} \mathrm{~d} z=h \frac{\partial p}{\partial x}, \\
& \int_{0}^{h} \frac{\partial^{u}}{\partial z^{2}} \mathrm{~d} z=\left.\frac{\partial u}{\partial z}\right|_{z=h}-\left.\frac{\partial u}{\partial z}\right|_{z=0}=-\left.\frac{\partial u}{\partial z}\right|_{z=0} \\
& \int_{0}^{h}\left(\frac{\partial u}{\partial t}+u \frac{\partial u}{\partial x}+v \frac{\partial u}{\partial y}+w \frac{\partial u}{\partial z}\right) \mathrm{d} z=\int_{0}^{h}\left(\frac{\partial u}{\partial t}+\frac{\partial(u u)}{\partial x}+\frac{\partial(v u)}{\partial y}+\frac{\partial(w u)}{\partial z}\right) \mathrm{d} z \\
& =h\left(\frac{\partial \bar{u}}{\partial t}+\bar{u} \frac{\partial \bar{u}}{\partial x}+\bar{v} \frac{\partial \bar{u}}{\partial y}\right)+\frac{\partial}{\partial x} \int_{0}^{h}(\bar{u}-u)^{2} \mathrm{~d} z+\frac{\partial}{\partial y} \int_{0}^{h}(\bar{u}-u)(\bar{v}-v) \mathrm{d} z .
\end{aligned}
$$

The DAF of the $v$-momentum, Eq. (23) is derived in a similar fashion. After dividing through by the film thickness, the resulting governing equations are Eqs. (9) and (10) for the unknown averaged velocities $\bar{u}(x, y, t)$, $\bar{v}(x, y, t)$ and the film thickness $h(x, y, t)$. The DAF of the governing equations contains friction and dispersion terms, namely $\left.\frac{\partial u}{\partial z}\right|_{z=0},\left.\quad \frac{\partial v}{\partial z}\right|_{z=0}, \quad \int_{0}^{h}(\bar{u}-u)^{2}, \quad \int_{0}^{h}(\bar{v}-v)^{2}, \quad \int_{0}^{h}(\bar{u}-u)(\bar{v}-v) \mathrm{d} z$, these terms can be determined by assuming that the velocity profile within the film has the same and consistent self-similar form as the classical Nusselt solution, namely:

$u=3 \bar{u}\left(\xi-\frac{1}{2} \xi^{2}\right), v=3 \bar{v}\left(\xi-\frac{1}{2} \xi^{2}\right)$, 
where $\xi=z / h$. Using relations (30) and (34) then leads to the following analytical expressions for these terms:

$$
\begin{aligned}
& \left.\frac{\partial u}{\partial z}\right|_{z=0}=\frac{3 \bar{u}}{h}, \\
& \left.\frac{\partial v}{\partial z}\right|_{z=0}=\frac{3 \bar{v}}{h}, \\
& \int_{0}^{h}(\bar{u}-u)^{2}+\int_{0}^{h}(\bar{u}-u)(\bar{v}-v) \mathrm{d} z=\frac{1}{5}\left(h \bar{u} \frac{\partial \bar{u}}{\partial x}+h \bar{v} \frac{\partial \bar{u}}{\partial x}-\bar{u} \frac{\partial h}{\partial t}\right), \\
& \int_{0}^{h}(\bar{v}-v)^{2}+\int_{0}^{h}(\bar{u}-u)(\bar{v}-v) \mathrm{d} z=\frac{1}{5}\left(h \bar{u} \frac{\partial \bar{v}}{\partial x}+h \bar{v} \frac{\partial \bar{v}}{\partial x}-\bar{v} \frac{\partial h}{\partial t}\right) .
\end{aligned}
$$

The final form of the DAF equations, using these friction and dispersion terms are found in Eqs. (9)-(12).

\section{Appendix B. Axisymmetric DAF equations}

The axisymmetric version of the DAF equations leads to two extra inertial pressure terms in the governing DAF equations, as seen below:

$$
\begin{aligned}
& \mathscr{E} \operatorname{Re}\left[\frac{\partial \bar{u}}{\partial t}+\bar{u} \frac{\partial \bar{u}}{\partial r}+\frac{1}{5 h}\left(h \bar{u} \frac{\partial \bar{u}}{\partial r}-\bar{u} \frac{\partial h}{\partial t}\right)\right]=-\frac{\operatorname{Re}}{\mathrm{We}} \frac{\partial p}{\partial r}+\frac{3 \bar{u}}{h^{2}}, \\
& \frac{\partial h}{\partial t}+\frac{1}{r} \frac{\partial}{\partial r}(r h \bar{u})=0, \\
& p=-\frac{\mathrm{We}}{\mathrm{Fr}^{2}} \frac{1}{r} \frac{\partial}{\partial r}\left(\frac{r \partial h}{\partial r}\right)+\frac{\mathrm{We}}{\mathrm{Fr}^{2}}(h-z)-\frac{\mathrm{We}}{\mathrm{Fr}^{2}} \Pi(h),
\end{aligned}
$$

where $r$ is the radial coordinate.

\section{References}

1. Yarin AL, Weiss DA (1995) Impact of drops on solid surfaces: self-similar capillary waves, and splashing as a new type of kinematic discontinuity. J Fluid Mech 283:141-173

2. Sivakumar D, Tropea C (2002) Splashing impact of a spray onto a liquid film. Phys Fluids 14(12):L85-L88

3. Naber J, Reitz RD (1988) Modelling engine spray/wall impingement. SAE Trans 1:118-140

4. Roisman IV, Tropea C (2002) Impact of a drop onto a wetted wall: description of crown formation and propagation. J Fluid Mech 472:373-397

5. Moreira A, Moita A, Panao M (2010) Advances and challenges in explaining fuel spray impingement: how much of single droplet impact research is useful? Progr Energy Combus Sci 36(5):554-580

6. Vander Wal RL, Berger GM, Mozes SD (2006) Droplets splashing upon films of the same fluid of various depths. Exp Fluids 40(1):33-52

7. Yarin AL (2006) Drop impact dynamics: splashing, spreading, receding, bouncing. Annu Rev Fluid Mech 38(1):159-192

8. Šikalo Š, Tropea C, Ganić E (2005) Dynamic wetting angle of a spreading droplet. Exp Therm Fluid Sci 29(7):795-802

9. Bonn D, Eggers J, Indekeu J, Meunier J, Rolley E (2009) Wetting and spreading. Rev Modern Phys 81(2):739

10. Eral H, Oh J et al (2013) Contact angle hysteresis: a review of fundamentals and applications. Colloid Polym Sci 291(2):247-260

11. Šikalo Š, Marengo M, Tropea C, Ganić E (2002) Analysis of impact of droplets on horizontal surfaces. Exp Therm Fluid Sci 25(7):503-510

12. Roisman IV, Tropea C (2005) Fluctuating flow in a liquid layer and secondary spray created by an impacting spray. In J Multiphase Flow 31(2):179-200

13. Worthington A (1897) Impact with a liquid surface, studied by the aid of instantaneous photography. Philos Trans R Soc Lond A Math Phys Eng Sci 189:137-148

14. Bernardin JDJ, Stebbins CJ, Mudawar I (1996) Effects of surface roughness on water droplet impact history and heat transfer regimes. Int J Heat Mass Transfer 40(1):73-88 
15. Cossali G, Brunello G, Coghe A, Marengo M (1999) Impact of a single drop on a liquid film: experimental analysis and comparison with empirical models. In: Italian Congress of Thermofluid Dynamics UIT, Ferrara vol 30, pp 1-12

16. Rioboo R, Bauthier C, Conti J, Voue M, De Coninck J (2003) Experimental investigation of splash and crown formation during single drop impact on wetted surfaces. Exp Fluids 35(6):648-652

17. Rioboo R, Marengo M, Tropea C (2002) Time evolution of liquid drop impact onto solid, dry surfaces. Exp Fluids 33(1):112-124

18. Šikalo S, Ganić E (2006) Phenomena of droplet-surface interactions. Exp Therm Fluid Sci 31(2):97-110

19. Macklin WC, Metaxas GJ (1976) Splashing of drops on liquid layers. J Appl Phys 47(9):3963-3970

20. Kim HY, Feng ZC, Chun JH (2000) Instability of a liquid jet emerging from a droplet upon collision with a solid surface. Phys Fluids 12(3):531-541

21. Rieber M, Frohn A (1999) A numerical study on the mechanism of splashing. Int J Heat Fluid Flow 20(5):455-461

22. Trujillo MF, Lee CF (2001) Modeling crown formation due to the splashing of a droplet. Phys Fluids 13(9):2503-2516

23. Weiss DA, Yarin AL (1999) Single drop impact onto liquid films: neck distortion, jetting, tiny bubble entrainment, and crown formation. J Fluid Mech 385:229-254

24. Cossali GE, Marengo M, Coghe A, Zhdanov S (2004) The role of time in single drop splash on thin film. Exp Fluids 36(6):888-900

25. Extrand CW (2002) Model for contact angles and hysteresis on rough and ultraphobic surfaces. Langmuir 18(21):7991-7999

26. Joanny J, De Gennes PG (1984) A model for contact angle hysteresis. J Chem Phys 81(1):552-562

27. Mao T, Kuhn DC, Tran H (1997) Spread and rebound of liquid droplets upon impact on flat surfaces. AIChE J 43(9):2169-2179

28. Scheller BL, Bousfield DW (1995) Newtonian drop impact with a solid surface. AIChE J 41(6):1357-1367

29. Fukai J, Tanaka M, Miyatake O (1998) Maximum spreading of liquid droplets colliding with flat surfaces. J Chem Eng Jpn 31(3):456-461

30. Roisman IV, Rioboo R, Tropea C (2002) Normal impact of a liquid drop on a dry surface: model for spreading and receding. Proc R Soc Lond Ser A Math Phys Eng Sci 458(2022):1411-1430

31. Guo Y, Wei L, Liang G, Shen S (2014) Simulation of droplet impact on liquid film with clsvof. Int Commun Heat Mass Transfer 53:26-33

32. Jacqmin D (1999) Calculation of two-phase Navier-Stokes flows using phase-field modeling. J Comput Phys 155(1):96-127

33. Lee SH, Hur N, Kang S (2011) A numerical analysis of drop impact on liquid film by using a level set method. J Mech Sci Technol 25(10):2567

34. Ming C, Jing L (2014) Lattice Boltzmann simulation of a drop impact on a moving wall with a liquid film. Comput Math Appl 67(2):307-317

35. Shetabivash H, Ommi F, Heidarinejad G (2014) Numerical analysis of droplet impact onto liquid film. Phys Fluids 26(1):012102

36. Krechetnikov R, Homsy GM (2009) Crown-forming instability phenomena in the drop splash problem. J Colloid Interface Sci 331(2):555-559

37. Mcguirk JJ, Rodi W (1978) A depth-averaged mathematical model for the near field of side discharges into open-channel flow. J Fluid Mech 86(4):761-781

38. Veremieiev S, Thompson H, Lee Y, Gaskell P (2010) Inertial thin film flow on planar surfaces featuring topography. Comput Fluids 39(3):431-450

39. Veremieiev S, Thompson H, Lee Y, Gaskell P (2011) Inertial two- and three-dimensional thin film flow over topography. Chem Eng Process Process Intens 50(5-6):537-542

40. Veremieiev S, Thompson H, Scholle M, Lee Y, Gaskell P (2012) Electrified thin film flow at finite reynolds number on planar substrates featuring topography. Int J Multiphase Flow 44:48-69

41. Veremieiev S, Brown A, Gaskell PH, Glass CR, Kapur N, Thompson HM (2014) Modelling the flow of droplets of bio-pesticide on foliage. Interfacial Phenom Heat Transfer 2(1):1-14

42. Ruyer-Quil C, Manneville P (1998) Modeling film flows down inclined planes. Eur Phys J B Condens Matter Complex Syst 6(2):277-292

43. Shkadov VY (1967) Wave flow regimes of a thin layer of viscous fluid subject to gravity. Fluid Dynam 2(1):29-34

44. Shkadov VY (1968) Wave-flow theory for a thin viscous liquid layer. Fluid Dynam 3(2):12-15

45. John V, Rang J (2010) Adaptive time step control for the incompressible Navier-Stokes equations. Comput Methods Appl Mech Eng 199(9-12):514-524

46. Adams MF (2012) A low memory, highly concurrent multigrid algorithm. arXiv Numerical Analysis

47. Gaskell P, Jimack P, Sellier M, Thompson H (2004) Efficient and accurate time adaptive multigrid simulations of droplet spreading. Int J Numer Methods Fluids 45(11):1161-1186

48. Lee Y, Thompson H, Gaskell P (2007) An efficient adaptive multigrid algorithm for predicting thin film flow on surfaces containing localised topographic features. Comput Fluids 36(5):838-855

49. Thompson M, Ferziger JH (1989) An adaptive multigrid technique for the incompressible navier-stokes equations. J Comput Phys 82(1):94-121

50. Chou SH, Kwak DY (2002) Multigrid algorithms for a vertex-centered covolume method for elliptic problems. Numer Math 90(3):441-458

51. Schwartz LW, Eley RR (1998) Simulation of droplet motion on low-energy and heterogeneous surfaces. J Colloid Interface Sci 202(1):173-188

52. Sui Y, Ding H, Spelt PD (2014) Numerical simulations of flows with moving contact lines. Annu Rev Fluid Mech 46:97-119

53. Starov V, Kalinin V, Chen JD (1994) Spreading of liquid drops over dry surfaces. Adv Colloid Interface Sci 50:187-221 
54. Savva N, Kalliadasis S (2011) Dynamics of moving contact lines: a comparison between slip and precursor film models. EPL (Europhys Lett) 94(6):64004

55. Sigmund O, Petersson J (1998) Numerical instabilities in topology optimization: a survey on procedures dealing with checkerboards, mesh-dependencies and local minima. Struct Optim 16(1):68-75

Publisher's Note Springer Nature remains neutral with regard to jurisdictional claims in published maps and institutional affiliations. 